\title{
Developing a multi-agent platform supporting patient hospital stays following a socio-technical approach: Management and governance benefits
}

\author{
Yves Wautelet $^{\mathrm{a}, *}$, Manuel Kolp ${ }^{\mathrm{b}}$, Samedi Heng ${ }^{\mathrm{b}}$, Stephan Poelmans ${ }^{\mathrm{a}}$ \\ ${ }^{a} \mathrm{KU}$ Leuven, Belgium \\ ${ }^{\mathrm{b}}$ Université catholique de Louvain, LouRIM, Belgium
}

\section{A R T I C L E I N F O}

\section{Keywords:}

Healthcare processes

Intelligent healthcare systems

Multi-agent system

Socio-technical approach

Pervasive healthcare

\begin{abstract}
A B S T R A C T
Most of the caregivers working in hospitals are highly skilled and educated work force. Even if they are supported by administrative staff, part of their time still consists of administrative procedures or trying to empirically fill or retrieve agendas on the basis of real-time constraints. Similarly, patients waste time waiting for adequate treatments so they occupy slots (beds) in hospitals while their hospitalization is not a necessity. This leads to huge wastes of resources for the hospital that has to hospitalize and prioritize patients in more effective need of care and monitoring. In order to ensure real-time patient support during its entire stay in the hospital (in terms of bed occupancy, appointments with doctors and/or for medico-technical exams) as well as to furnish a central solution open for future IT integration we propose, in this paper, to build a Multi-Agent System (MAS) based software solution. The latter has been built following a sociotechnical approach; it maps, at runtime, the working processes of a Belgian hospital. Processes have been modeled in a patient-centric way in order to identify most relevant stages and bottlenecks in bed, cares and medico-technical exams management; real-time data transmission allows to update and re-optimize slots statuses as well as schedules. Also, data analysis (i.e., business intelligence) functions are supported by the MAS implementation for decision making/ taking. Further than the proof of concept that shows the managerial support of the software solution, the MAS modules' benefits to the overall hospital strategy is studied. The latter shows the relevance of the solution to the hospital's governance.
\end{abstract}

\section{Introduction}

Healthcare institutions are nowadays facing a lot of challenges. Among their strategic objectives, they primarily need to furnish high quality care to patients but also to, at least, break even from a financial point of view. Their mission to society is important and complex so that tools and procedures need to be optimized to furnish an adequate added value. One of the strategies that can be used in order to sustain this long term plan is the use of Information Technology (IT). For instance, (Yen and Bakken, 2012; Payne et al., 2013) highlight that IT is (notably) able to increase efficiency, quality, safety of medical care, data analysis, cost-effectiveness and fairness in healthcare.

Following (Varshney, 2009), pervasive healthcare is the conceptual system of providing healthcare to anyone, at any time, and anywhere

\footnotetext{
* Corresponding author.

E-mail addresses: yves.wautelet@kuleuven.be (Y. Wautelet), manuel.kolp@uclouvain.be (M. Kolp), samedi.heng@uclouvain.be (S. Heng), stephan.poelmans@kuleuven.be (S. Poelmans).
} 
by removing restraints of time and location while increasing both the coverage and the quality of healthcare. Various IT technologies supporting treatments or healthcare processes can support this system. In order to furnish their full potential, these technologies need to be successfully integrated in the hospital environment but also need to be easily integrated with other technologies and be scalable (Cresswell et al., 2017). With more and more connected objects available at an ever decreasing price, the amount of heterogeneous technologies possibly of benefit for healthcare services will continue to grow leading to an even bigger challenge for (pervasive) healthcare.

Currently used software systems in hospitals are often too old and closed to ensure software collaboration/integration (Bergenti and Poggi, 2010). Acting as IT consultants and researchers, the authors have studied the processes and IT infrastructure of a Belgian hospital. At the start of the research, the information systems of this hospital were obsolete and not coupled nor integrated. These systems were connected with a central database coming from the reorganization of multiple other (previous) datastores having different purposes and linked together in a "do it yourself" way, a common problem in healthcare institutions notably highlighted by Bergenti and Poggi (2010). It was thus poorly conceived and not fully normalized so that history and consistency were not ensured. A new normalized and centralized database needed to be developed from scratch; the latter would be the central point where data is stored in a standard and unified way and will constitute a potential source for data analysis. Moreover, previous information systems only supported parts of the processes of the hospital so that a lot of work was still done manually. All this has negative consequences on the fulfillment of the hospital long term strategy. The governance board consequently wished for a new integrated IT system notably offering openness for later evolution to connected technology, the highest possible process support but also allowing exploitation of the data for business intelligence. Globally, The project was seen as an opportunity to reach a higher alignment between the hospital's business objectives and the supporting IT infrastructure.

Facing all these challenges, we have decided to develop a central software platform following a socio-technical approach (see Davis et al., 2014). The software development, within its first increment, targets the support of the bed management process of the hospital. All the roles involved in the hospital work progress are stakeholders of this process and it constitutes their means of coordination. Bed management has been for a long time identified by the various hospital services and Care Units ( $C U$ ) as the main bottleneck for a global optimization of the entire set of operational processes (see for example Hall (2012); Shi et al. (2015); Maloney et al. (2007)); it constituted the root cause of inefficiency in the hospital: e.g., unoccupied beds, transfer to other hospitals when beds are not available, patients waiting in their bed for medical exams during prohibitive delays, etc. No place must be wasted in order to avoid financial loss but simultaneously the services offered must be kept at highest standards since the stake is life and death. Global realtime coordination and analytics must be offered for this critical business process to increase the overall managerial efficiency and progress towards the overall strategic objectives. Also, future connected devices (e.g. beds) are likely to furnish more data potentially useful for planning. These devices must be easily integrable in the coming software system.

The objective of the paper is not to document runtime processing like scheduling algorithms but to show how a socio-technical approach can be followed to develop an IT system aligned with, on the managerial side, the hospital operational processes, and, on the governance side, the organizational strategy. The software development stages (analysis, design and implementation) presented mainly focus on the processes aiming the optimal repartition of patients in the slots (i.e., the beds) of the various CUs; the followed modeling and design approaches are very representative of how IT can be aligned within the organization. We call this particular software module the bed planner. Other aspects like the management and analysis of appointments both for hospitalizations, simple (doctors) consultations or medico-technical exams (called the Medico Technical Exams Management App), the management and analysis of the medical and administrative files of the patients (called the Care Unit Management App) and the automatic billing of the patient medical services (called the Billing Processor) are also part of the core processes supported by the platform but are not the focus of the present presentation. The scientific approach and validity (in order to answer the research question) is nevertheless the same for these other aspects because they have been built following the same socio-technical approach and evaluated with the same tools.

The main contribution of the paper is thus to show how a Multi-Agent System (MAS) based platform mapping the behavior of the hospital processes at runtime can be built using a socio-technical approach. Such an approach leads to operational benefits, an IT system mapping, at runtime, the behavior of the real life organizational processes. We also mention it leads to building a centralized database that can be later exploited for datawarehousing as well as the identification of the key elements for data analysis. It finally leads to governance benefits, through an alignment with the long term organization's strategic objectives.

The processes modeled to develop the software solution are centered on the patient. It is indeed the key stakeholder to be satisfied for bed management. The analysis provided in this paper mostly focuses on tracing the benefits of the platform to the management and governance of the hospital. As far as governance is concerned, the overall long term strategy of the hospital has been depicted in terms of goals so that the contributions of the software modules on these goals could be traced. In line with the special issue theme, the economic, social and cultural benefits of the platform are notably highlighted.

The paper is structured as follows:

- Section 2 depicts the research question, research method and the data collection. The aim of the collected data is to model the hospital as a socio-technical organization and to build a MAS out of it;

- Section 3 exposes the technical choices for the development of the software solution;

- Section 4 positions the paper with respect to previous scientific work and contributions;

- Section 5 relates the most important stakeholders for ensuring pervasive healthcare through agent software supported bed management;

- Section 6 exposes the modeling of organizational processes for interfacing with the patient using the $i^{*}$ socio-intentional framework (Yu et al., 2011). This modeling of the current situation allows to build a complete and consistent basis for positioning the 
development of the software services;

- Section 7 depicts the modeling of the decomposition of the bed reservation process using the strategic rationale diagram approach;

- Section 8 overviews the functions of the software system-to-be are depicted using the same strategic rationale diagram approach. The application of this framework allows the reengineering of the process for a more optimal and fully IT supported realization. This is notably allowed by the increase of information available and transmitted to the central database;

- Section 9 explains the MAS architecture and detailed design;

- Section 10 is devoted to the MAS implementation. A prototype of the MAS-based system is presented in the paper; it aims is mainly to illustrate what the platform is rather than to demonstrate/show each of the functions;

- Section 11 relates the modeling of the long-term hospital strategy and the study of the added value of the MAS-based platform in terms of operational management and business intelligence capabilities on this strategy. Economic, social and cultural benefits are highlighted;

- Section 12 discusses the impact of the research on the hospital patient-centric approach and provides a SWOT analysis of the MASdevelopment project;

- Section 13 finally concludes the paper.

\section{Research question, research method and empirical data collection}

\subsection{Research question}

We here define the main (research) question we aim to answer: What are the management and governance benefits of a MAS-based platform developed using a socio-technical approach?

Management benefits are the support and central coordination of the hospital bed management processes. In our socio-technical approach, we start from advanced organizational models of the hospital in order to design and implement a MAS as much as possible aligned, at runtime, with the organization. The modeling process is centered on the actors - notably the patient - and aimed to integrate the to-be software system as smoothly as possible in the existing organization.

More managerial benefits include the use of the data centralized by the use of the unique MAS-based platform for business intelligence. The socio-technical approach allows to identify key activities, resources, bottlenecks, etc. where information should be generated for a better understanding of the organizational performance.

Governance benefits include the support of the new MAS-based software solution on the organization's (long-term) strategic objectives. To this end, a modeling of the hospital long term goals has been realized and represented with the Non-Functional Requirements (NFR) framework (Chung et al., 2000). It can be shown that the socio-technical approach followed lead to the development of a MAS platform offering various positive impacts on these goals.

The steps of this research process are detailed in the next section.

\subsection{Research method}

The research presented in this paper has followed a Design Science Research (DSR) methodology for Information Systems. DSR involves the creation of new knowledge through design of novel or innovative artifacts (things or processes) and analysis of the use and/or performance of such artifacts along with reflection and abstraction to improve and understand the behavior of aspects of Information Systems (Ulrich, 2011). Typically, design sciences attempt to create things that serve human purposes, not to understand reality as in natural sciences (Simon, 1996). Similarly, the research conducted in this paper is aimed to provide a useful tool (in the form of the MAS based-platform) to the hospital stakeholders. Roughly speaking, this particular methodology synthesizes previous DSR Methodologies in IS such as Peffers et al. (2007) or Hevner et al. (2004). Furthermore we emphasize that our research is objective-driven. This means that the original start of the research process is the definition of the objectives of the solution to provide: an open MAS-based platform supporting the hospital processes and positively contributing to organization long-term strategy. In the rest of this section, we align each of the DSR steps with the instantiation that we have made of it in this study.

More specifically, we followed the methodology of Peffers et al. (2008) consisting of 6 steps:

- Problem identification and motivation. The problem identified is mainly the inefficiency of the existing bed management (see Section 1) solution coupled with the evolution of healthcare-based technologies. Indeed, with the ubiquitous presence of IT and connected devices and the pressure for pervasive healthcare, open and flexible software systems are required for process optimization and to ensure proper IT integration;

- Definition of the objectives for a solution. The objectives are to build a MAS-based software platform to support the bed management processes and studying its alignment with the hospital long term strategy;

- Design and development. The required empirical data collection is depicted in Section 2.3. Also, the methodological and technological choices for the development of the platform are exposed in Section 3. Basically, we highlight here that the Tropos software engineering methodology (Castro et al., 2013; Castro et al., 2002) was used to build the MAS based platform. Tropos uses $\mathrm{i}^{*}$ diagrams at its early and late requirements stages so that various $\mathrm{i}^{*}$ diagrams have been built to represent the hospital as an organization made of multiple dependent actors. Also, to represent the dynamic of the processes of the Bed Reservation Service (BRS), BPMN Business Process Diagrams (OMG, 2013; Chinosi and Trombetta, 2012; Stephen, 2008) have also been used. All of these diagrams are original contributions of this paper and are part of a larger models set; the ones presented here focus on bed 


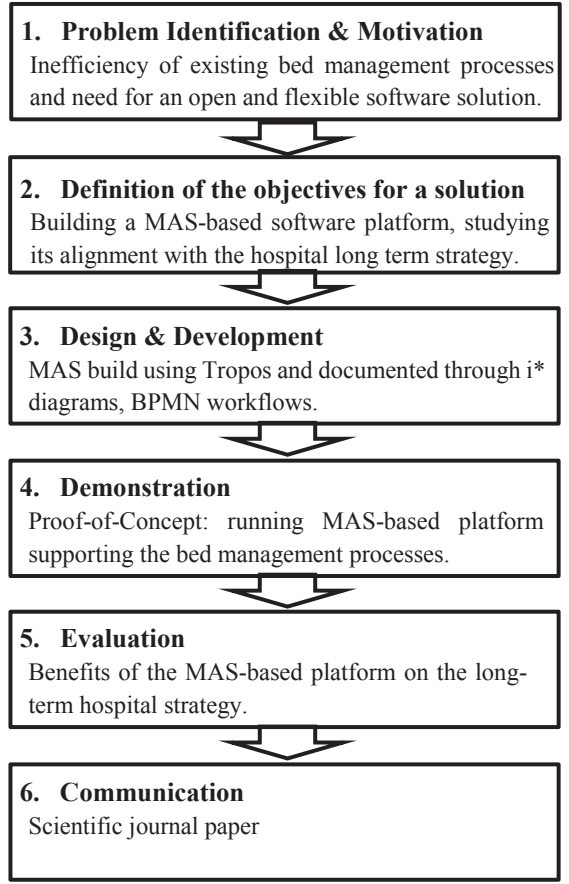

Fig. 1. Design Science Research Methodology for Information Systems: Acquisition architecture.

reservation to build the Bed Planner module. These models are built in order to be easily transformed into a MAS design. We also note that, for the MAS architectural design, the joint venture architectural style (see Kolp et al., 2008) has been used. In addition to classical architectural styles, software literature has defined organizational architectural styles (see e.g., Tamburri et al., 2013) for cooperative, dynamic and distributed applications like multi-agent systems. The joint-venture style is a generic one (so not health specific) that has been adopted in various domains like for example education (see Kolp and Wautelet, 2015; Wautelet et al., 2016). Criteria and procedure for architecture selection are given into (Kolp et al., 2011); it was the most relevant one to be used in the present context. Also, the Jadex agent platform (Braubach et al., 2003) has been used as implementation language;

- Demonstration. The demonstration stage involves showing the ability and efficiency of the MAS-based platform to support the bed management processes. This work is depicted in details in Sections 9,2,3,4,5,6,7,8,9,10.

- Evaluation. The MAS-based platform has been evaluated with respect to the long-term hospital strategy. The latter has been modeled through the interviews of the hospital governance board. The evaluation is realized in Section 11.

- Communication. The main communication has been done in the present paper.

The acquisition architecture instantiated to our research can be found in Fig. 1.

\subsection{Data contextualization}

Empirical data about the hospital processes and the IT issues have been collected as primary input to our research. All the data used for the building of the MAS-platform was qualitative; it has mostly been collected through interviews. This has not been realized as a one shot activity but rather as a continuous process, more precisely:

- The modeling of the as-is hospital processes using the $\mathrm{i}^{*}$ modeling framework as well as BPMN has been conducted on the basis of interviews of people playing the various roles within the organization as well as through the analysis of (informal) existing workflow documents (the result of this first collection of empirical data can be found in $A, B, C, D^{1}$ ). More details can be found in Wautelet (2017). A first set of interviews allowed to produce a first set of models then a second set of interviews allowed to clarify some elements and finally a validation of the produced models has been realized through a workshop with people of the various roles involved. The data collection process not only focused on existing procedures but also included collecting advices, desiderata and other forms of requirements of all of the stakeholders useful to guide the building of the to-be situation. All the data collected here was unstructured and in natural language;

- The identification of the existing IT infrastructure has been done through the analysis of technical documents furnished by the IT department as well as through interviews of the IT staff. The data collected here includes unstructured data but also former

\footnotetext{
${ }^{1}$ To be readable, the collected data as presented has been structured in the appendix.
} 
database models with various degree of normalization;

- The identification of inefficiencies has led to processes re-engineering and the definition of the software system-to-be implemented as a MAS. It has been driven by a classical engineering approach of transforming requirements and organizational models to a MAS implementation, namely Tropos;

All in all, a face-to-face contact with the stakeholders has always been privileged over online communication means. People playing each of the relevant roles have been continuously available to clarify the various questions of the modeling team so knowledge has been built in an iterative way.

\section{Justification of the technical approach}

A hospital is, today, a socio-technical organization consisting of humans and machines interacting to provide the best care to the patients. It can be seen as a complex system involving technical devices where various caregivers and administrative staff all organized in independent entities interact with each other and with patients to furnish the best possible service (Pickering et al., 2012). Each patient nevertheless will have a genuine experience of its hospital stay. Patients go from one unit to the other following their caring needs and each of them has a personalized journey depending on the nature of his/her ills and the way he/she reacts to treatments. As mentioned, series of actors are involved in these various hospital processes and various information systems with lots of heterogeneous data sources produced by humans and other (possibly smart) devices spread over the entire organization are used. To ensure a satisfying patient experience in such an environment, software systems require to furnish real-time tailored support through coordination and/or integration.

In order to build such software in complex socio-technical environments, intelligent agent technology provides various kinds of benefits. Agents are virtual entities taking autonomous actions upon their environment in order to achieve individual or collective goals. Moreover, these agents are capable of learning to improve the performance of their decisions in time. Classical computational paradigms (like for example object-oriented) are limited when modeling complex settings (Fox et al., 2003) because they are driven by models based on concepts from computer systems rather than organizational and intentional oriented ones (Kolp and Wautelet, 2015; Hogg and Jennings, 2001).

As said, today's hospitals are made of hundreds of different types of elements generating data; these can be seen and conceptualized as agents: e.g., humans, software agents, medical machines, automates, sensors, electrocardiograms, thermometers, controllers, mobile devices, scanners, etc. These are agents in the sense that:

- they are autonomous and dedicated to specific tasks;

- they are situated in a physical environment;

- they can act upon their environment if this is necessary by warning users, proposing solutions or taking autonomous action.

Multi-Agent Systems (MAS) (Wooldridge, 2009) can bring coordination of healthcare processes and these heterogeneous devices to a higher level. MAS can then deliver their inherent qualities like flexibility, dynamics and reliability to medical software systems (Isern and Moreno, 2016; Isern et al., 2010). Therefore, this paper reports on the research aimed to build a MAS-based platform for the management of the patient stay in the hospital. By mapping the behavior of the hospital actors at run-time through the collection of data from various sources, this MAS-based platform allows (among other functions) a (real-time) dynamic (re) allocation of bed occupancy, doctors and medico-technical exams planning and automatic invoicing of healthcare services. This especially avoids wastes and allows to strictly identify each care or medico-technical exam that the patient benefits from more efficient accounting. The development of the MAS-based platform in the hospital allows to reduce at maximum human intervention and, through the flexibility and openness, will allow to integrate new devices in the form of agents to furnish real-time data and lower the need for human intervention. Finally, the MAS can also be used as a business intelligence tool for decision support.

When modeling socio-technical environments, classical UML models like use case diagrams and activity diagrams (OMG, 2015) tend to be too limited in their representation abilities to capture social interactions and dependencies useful for understanding the software problem in global and particular terms (Yu, 2009). Important pieces of the problem which could be crucial when developing MAS-based software could thus be lost. That is why, MAS are mostly not developed by starting from traditional (object) modeling techniques and require their own supporting frameworks. The $\mathrm{i}^{*}$ framework is used here at modeling stage and, on a more global scale, follow the life-cycle of the Tropos software engineering methodology. Tropos adopts ideas from requirements engineering and organizational modeling where agents/actors and goals have been used heavily for requirements analysis, as well as transformation to MAS technologies, to define the architectural design and implementation phases. Tropos uses $\mathrm{i}^{*}$ models which offer actors (agents, roles or positions), goals, and actor dependencies as primitive concepts for modeling an application during requirements or

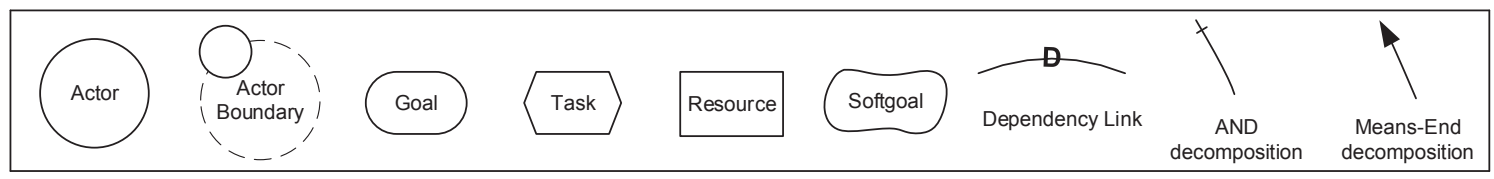

Fig. 2. $i^{*}$ Elements and their Graphical Representation. 
organizational/social analysis; they are thus better adapted when modeling complex organizations than for example UML use-case and activity diagrams. Fig. 2 distinguishes the main $i^{*}$ elements and their graphical representation used in the diagrams of the paper.

Further integration of our MAS-based platform with all kinds of hospital processes as well as with other kinds of medical applications using physical devices and connected objects will thus be facilitated through the use of agent technology. Ultimately, the integrated information system build-up using the Tropos approach allows to trace the full journey of the patient towards the hospital and assist at maximum all the administrative tasks of the medical staff as well as to generate each of the required administrative documents (invoice, medical care reimbursement documents, ...). Illustrating this nevertheless remains out of the scope of the paper that focuses on bed management.

Let us finally note that Tropos was proposed in two journal papers (i.e., Castro et al., 2002 and Bresciani et al., 2004) but was not fully implemented into some real-life software development environment. As a side effect, the paper also contributes to the discipline of agent-based software engineering to demonstrate practical suitability.

\section{Related work and positioning}

Isern and Moreno (2016) study many possible use of agent-based technologies in healthcare through a systematic state-of-the-art analysis on the application of agents in this domain; the paper suggests a way of clustering the contributions found. Their classification of "agent-based contributions" is built on an exhaustive literature review with a double objective: firstly, it is based on three possible types of users intended to use the MAS-based application and, secondly, on seven different types of such applications. The framework is consistently built and adequately covers existing literature so that it is used for positioning the contributions. More specifically, their structure is used here to overview the type of users targeted by the MAS-based platform developed in our case study (Section 4.1) and to define its type (Section 4.2).

\subsection{Positioning with respect to the User Base}

As evoked, Isern and Moreno (2016) firstly distinguish three types of usage in healthcare MAS-based applications; i.e., (i) patientcentered; (ii) staff-centered; and (iii) healthcare organization-centered. The platform developed in our case study is discussed with respect to these three types.

Patient-centered applications are devoted to bring particular services to the patient. Within our platform, the patient is at the center of the modeled, supported and analyzed business processes but is not a user of it. The contribution of this paper can thus not been described as a patient-centered application in the sense defined by Isern and Moreno (2016).

Staff-centered applications are designed to support caregivers in their daily activities. Isern and Moreno (2016) insist that these are mostly built as personal assistants for caregivers and their aim is to provide appropriate medical data helping these in specific medical circumstances. For instance, (Bouzguenda and Turki, 2014) builds up a MAS architectural style for dynamic medical crossorganizational workflow management. In short, it allows to link patient data over multiple systems located at different geographic places and present in different workflows. The coordination of these workflows is ensured by the coordination of software agents. This principle is also used in our approach, limited to stakeholders of a single organization but with more specific behavior. Liu et al. (2012), Liu et al. (2012), Chen et al. (2012), Chen et al. (2012) build a MAS to integrate heterogeneous and dispersed medical records that doctors can use for taking medical decisions. Our MAS adopts the same approach but goes much further ensuring global stakeholder coordination through the use of the MAS. A combination of the two previous approaches is thus adopted in this paper. Next to these approaches, other ones are devoted to use a MAS approach to build a specialized Decision Support System (DSS) from heterogeneous data sources (e.g. Sayyad-Shirabad et al., 2012; Taboada et al., 2011). Building such a DSS with our MAS approach is not the main goal of this paper.

Healthcare organization applications are targeted to all the stakeholders in the healthcare organization; this is precisely the first aim of our MAS-based platform. The modeling of the patient processes provides an ontological representation of the stakeholders and their dependencies so that, in terms of importance, the entire healthcare organization user base is involved. These stakeholders and their dependencies are studied in Section 5. The MAS is indeed intended to align at runtime with the overall hospital processes in order to optimize beds availability. Such an approach has been used in various application domains like particular diseases (Isern et al., 2012) or entire healthcare platforms (Bouchoul and Mostefai, 2009; Bhat et al., 2011; Nguyen et al., 2009).

\subsection{Positioning with respect to the type of application}

Besides the user-based classification, another one is proposed in Isern and Moreno (2016) based on the type of the application itself; i.e. (i) Data-management systems; (ii) Implementation of secure platforms; (iii) Decision support systems; (iv) Planning; (v) Simulation; (vi) Care platforms and (vii) Monitoring and alarms. This section highlights and positions our contribution with respect to these different application domains.

Data-Management Systems incorporate the systems focusing on the retrieval and processing of medical data. As evoked earlier, all the data concerning the patient and generated along the medical process is centralized and available for operational management of the patient processes. Cardoso et al. (2014), Cardoso et al. (2014), Cardoso et al. (2014) study the Agency for Integration, Diffusion and Archive of Medical Information (AIDA), which is an intelligent, agent-based platform to ensure interoperability in healthcare units. Agent communication is done through ACL messages (see e.g., Bellifemine et al., 2007) in the same way of the MAS described in this paper. It, however, does not have the same kind of interoperability issues as Bellifemine et al. (2007) because it integrates each aspect 
of the agent platform in the same technology.

Implementation of Secure Platforms concerns the ex-post integration of security measures to MAS-based platforms (see e.g., van Deursen et al., 2013). The present research is not focused on that particular aspect; security requirements have been taken into account but are out of the scope of the paper.

Decision Support Systems encompass systems devoted to support medical decisions on the basis of data gathered along healthcare processes. In line with Isern et al. (2012), the present research models the patient workflows for a later support through a MAS. Through this, significant amounts of data on the patient is gathered and crossed with third party databases for decision support. This aspect is nevertheless a side aspect of the main paper focus.

Planning systems encompass the coordination and scheduling of human and material resources under defined constraints (see e.g., Chennaoui and Paquet, 2014; Braubach et al., 2014). This is a fundamental aspect of the developed contribution that notably aims to globally optimize the patients' bed occupation.

Simulation encompass applications that simulate diseases through MAS. This kind of simulation is outside the scope of our contribution.

Care Platforms encompass healthcare-based platforms allowing to simulate healthcare departments such as emergency units. This is precisely the goal of our MAS-based platform that focuses on mapping the behavior of actors in the CU at runtime.

Monitoring and Alarms encompass applications that monitor the state of the patient. This is a completely different scope than ours.

\subsection{Related sources on the development of a MAS-based management platform}

As evoked the MAS-based platform is destined to support and automate bed reservation and occupancy procedures as a central mean of coordination for the hospital. The main focus of this paper is thus not to integrate an algorithmic approach of the problem like in Belciug and Gorunescu (2015); Braubach et al., 2014 but rather an organizational and process oriented one. Nevertheless the agents are able to organize and coordinate to furnish an optimal service and new algorithms can be included at will by adapting the agent beliefs base (see Sections 9 and 10).

A MAS-based mobile health care monitoring system is porposed in Gund et al. (2011). Its aim is to enhance communications between the hospital stakeholders by mapping hospital behavior at runtime. Their approach is comparable to ours in the sense that they follow an organizational model of the caring problem, they also aim to aggregate various sensors, systems, etc. through the agent approach and develop analytics from the collected data. Nevertheless the scope of their solution is much more limited than ours leading to very basic organizational models principally built up as an integration architecture. The solution described in this paper can be seen as a large scale enhancement of theirs and driven by bed management as a key process in the overall healthcare coordination.

A multi-agent software prototype to simulate the activities and roles inside a health care system is developed in Zhang et al. (2009). The prototype is developed using a framework called Role-based Agent Development Environment (RADE). The paper illustrates the design of the MAS through roles and the automatic agent generation process. A rule-based coordination approach is also proposed and illustrated with a runtime scenario. Similarly to Gund et al. (2011), the paper's approach is focused on creating a simple organizational scenario aligned with real life healthcare processes for agent coordination at runtime. Also, the agent model implemented at runtime is goal oriented. Our approach is nevertheless more global and not only focused on individual tasks but rather on the coordination of the entire bed management processes.

The MediMAS prototype, an implementation of a MAS-based solution mapping the behavior of a laboratory from a local Swiss hospital, is built in Nguyen et al. (2009). The MAS works on behalf of human agents, taking care of routine tasks, and thus increasing the speed, the systematic, and ultimately the reliability of the information exchanges. Authors claim that the modeling of the software agent layer can be methodically derived from the actual classical laboratory organization and practices, as well as seamlessly integrated with the existing information system. Their approach is very similar to ours but limited to a laboratory solution which does not integrate the most important processes of coordination in a hospital. Indeed, the patient is not involved in their solution which is thus completely asynchronous of the rest of the hospital processes.

Bouchoul and Mostefai (2009), Bouchoul and Mostefai (2009) examine the importance of Healthcare information systems (HCIS) integration and introduces MOBIFLEX, a generic architecture for integrating HCIS. The architecture of MOBIFLEX combines mobile agents, static agents and agents-services. The aim of the combination of these technologies is to ease flexible integration and exploitation of disparate HCIS sub-systems. A prototype of the system is built in JADE. Their approach is similar to ours in the adoption of heterogeneous systems and data sources. However, their system is limited to integration only and does not support any management process of the hospital.

As a summary the present research integrates the best of two approaches that are generally seen as independent in literature. The first is to focus on the organizational problem in order to have a core MAS that manages the bed reservation process seen as the main bottleneck in the entire hospital processes. The second is to integrate various systems as agents to share common data and allow global coordination.

\section{Organization of the hospital stakeholders}

This section briefly defines and describes the different roles played by individuals or groups of individuals in the hospital and highlights the interactions between these different roles. 


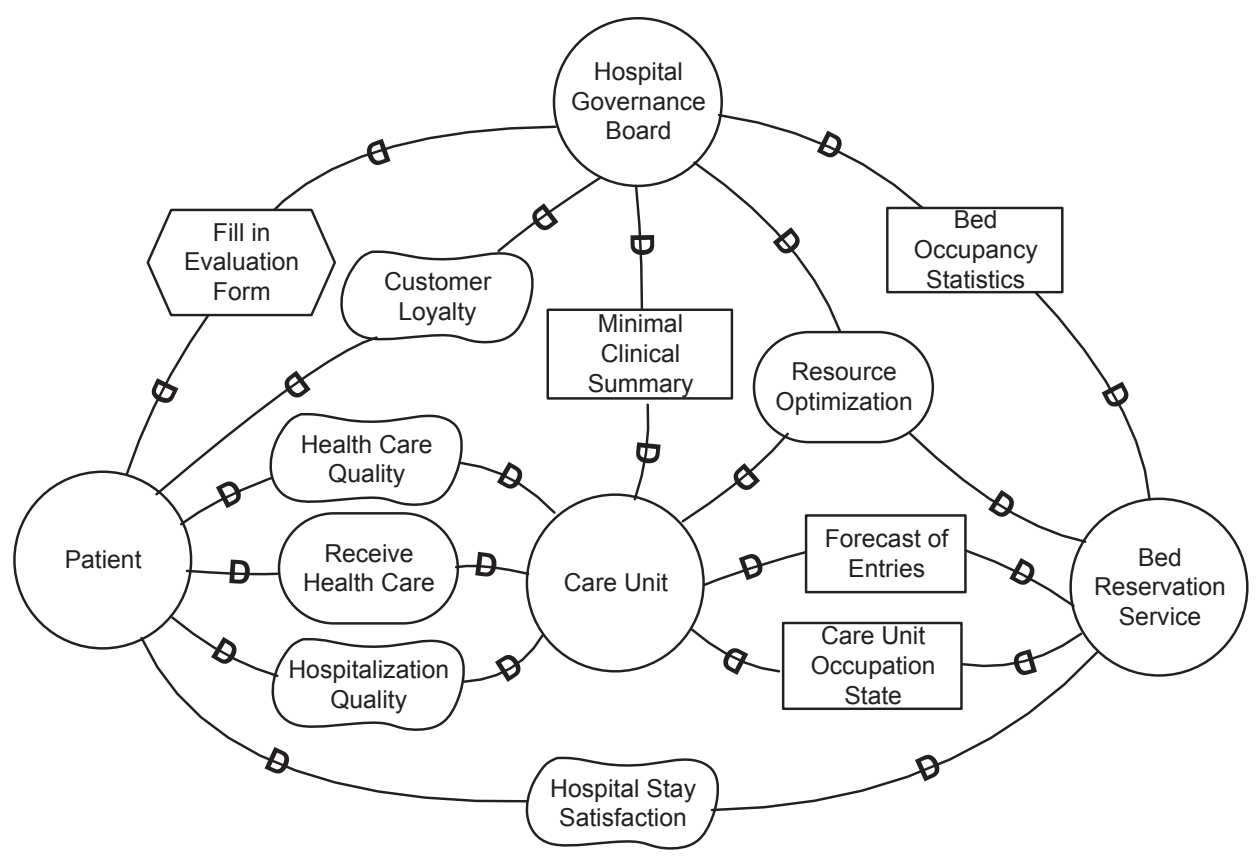

Fig. 3. Organizational Setting of the Bed Reservation Process.

A more detailed description of the roles can be found in A or Wautelet (2017). We succinctly distinguish here:

- The Patient is an individual receiving or registered to receive medical treatment. This role is crucial since the hospital's main purpose is to provide him care;

- The Care Units $(C U)$ are the departments of the hospital. Physically, a CU is made of a set of (bed) rooms where patients reside in order to get services around a specialty (for example oncology, pediatrics, stomatology, reanimation, etc.);

- The Healthcare Team provides the patients with medical care. It is mainly composed of nurses and doctors. Doctors are specialized into defined medical fields and their related treatments;

- The Administrative Services group several services. The admission is made of a set of desks where the patients need to register when they enter the hospital. The financial service manages the invoices and the entire financial management of the hospital. The patients' quality service monitors the clients' satisfaction with the hospital services (for instance by administering surveys);

- The Bed Reservation Service (BRS) directly organizes all the aspects of the stay of a hospitalized patient (bed reservation, booking of medico-technical treatments and consultations, etc.);

- The Administrative Nurse Assistant (ANA) is in charge of the interaction of the CU with the software system more particularly for encoding bed occupation in the CU. An ANA thus works within the range of a determined CU and there is one and only one ANA per service (see below). ANAs notably update the bed occupancy, the evolutions in the patient file, etc. They only perform administrative activities related to the bed occupancy;

- The Hospital Governance Board governs the hospital at its highest level and thus elaborates and puts in practice the strategies.

\section{Organizational analysis for interfacing with the patient (AS-IS situation)}

In the Tropos software engineering method, requirements analysis focuses on the intentions of stakeholders. Their intentions are modeled as goals which, through some form of a goal-oriented analysis, eventually lead to the functional and non-functional requirements of the system-to-be.

Specifically, for analyzing the problem of the Patient Hospital Stay, Fig. 3 models the organizational setting of the bed reservation business process using an $\mathrm{i}^{*}$ Strategic Dependency (SD) diagram ${ }^{2}$. At the strategic level, this reservation process implies four main actors (i.e., stakeholders) - Hospital Governance Board, Patient, Care Unit and Bed Reservation Service - depending on each other. The main constraints represented by goals dependencies ensure to Receive Health Care and target Resource Optimization while taking into account trade-offs modeled as softgoals such as Customer Loyalty, Health Care and Hospitalization Quality or Satisfaction.

Once the main relevant stakeholders and their goals have been identified such as in Fig. 3, a strategic rationale model determines

\footnotetext{
${ }^{2}$ The SD is a graph in which nodes represent actors (or stakeholders), and the links represent dependencies. By combining nodes and links, a SD builds a network of dependencies that help to graphically represent the external relationships between actors (or stakeholders). There are directed arrows to link the depender, dependum and dependee. These arrows indicate the direction of the dependency, determining which actor is the depender and which is the dependee.
} 


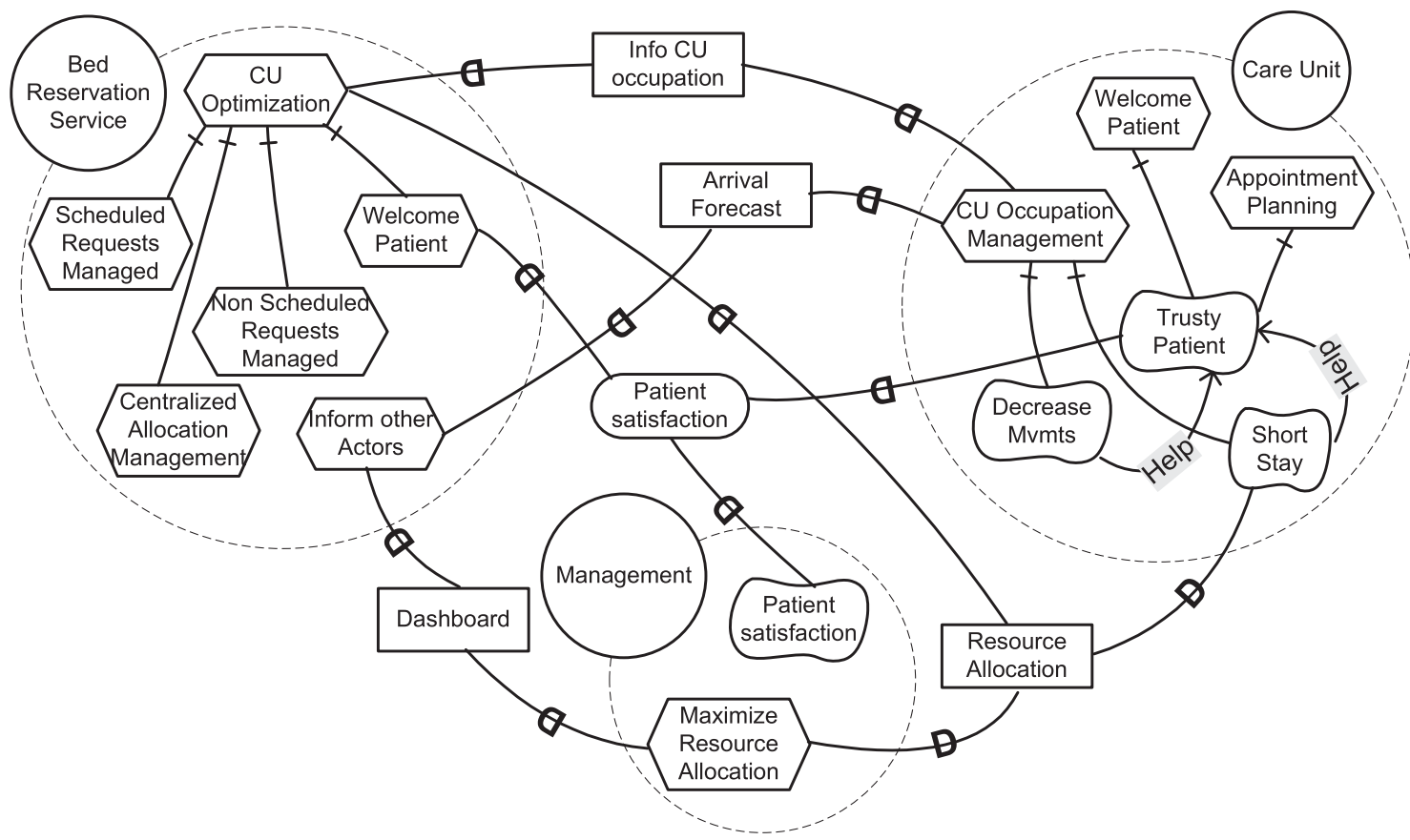

Fig. 4. Rationale Analysis of Care Unit and Bed Reservation Service.

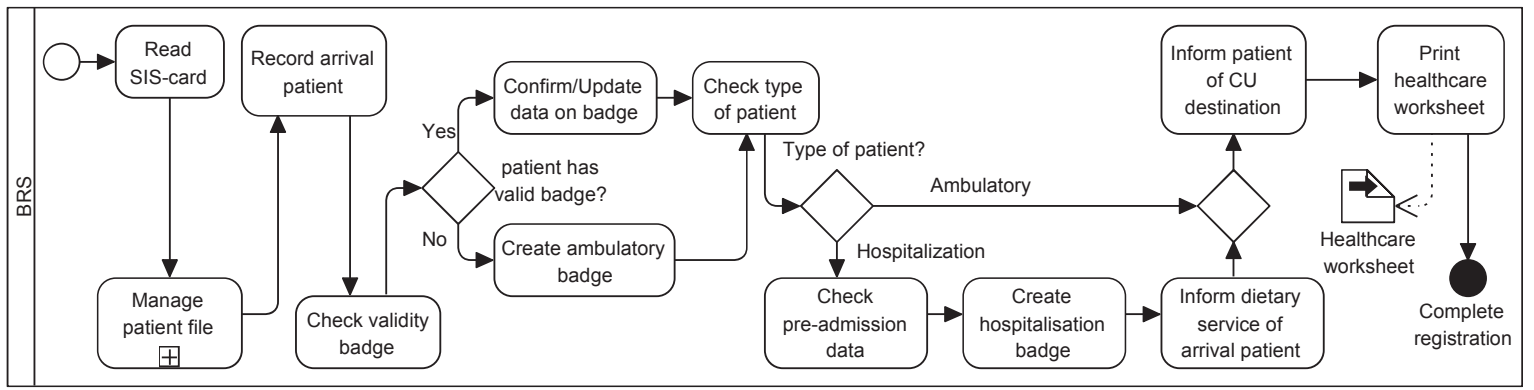

Fig. 5. Welcome Patient Workflow.

through a means-ends analysis how these goals (including softgoals) can actually be fulfilled through the contributions of other actors. An $\mathrm{i}^{*}$ strategic rationale model is used to this end. It is a graph with four types of nodes - goal, task, resource, and softgoal - and two types of links - means-ends links and task decomposition links. The graph captures the relationship between the goals of each actor and the dependencies through which the actor expects these dependencies to be fulfilled.

Therefore, Fig. 4 focuses on the goal dependency between actors Care Unit, Bed Reservation Service and Patient, namely Patient satisfaction. To achieve that goal, the rationale analysis postulates a task Welcome Patient and softgoals Trusty Patient and Patient Satisfaction realized and fulfilled by the above actors. Tasks are partially ordered sequences of steps intended to accomplish some (soft) goal. Tasks can be decomposed into goals and/or subtasks, whose collective fulfillment completes the task. In the figure, $C U$ Optimization is decomposed into more precise subtasks while $C U$ Occupation Management is refined by softgoals which together contribute to the top-level task.

Detailed analysis of tasks are specified in a classical way as processes using standard modeling notations such as OMG (2013). For instance, one of the processes, the Welcome Patient task, is specified as a workflow represented in Fig. 5. The original situation is as follows. The ANAs of the CU have to plan the stay of the patient one week before his/her hospitalization and also do the planning during his/her hospitalization. One week before the actual hospitalization of the patient, the ANA checks if there are still appointments to make that are not yet made by the BRS. This work is done by the ANA that crosschecks, on the one side, the bed reservation notes and hospitalization demands he/she receives from the BRS with, on the other side, the patient's file. If there are still some appointments missing, the ANA contacts the BRS to make them. Then, when the patient arrives, the ANA follows-up the planned treatments of the patient within the current CU. After these planned treatments, the ANA checks if the patient needs extra treatments or not. If the needs extra treatments within another CU, the ANA redirects the patient accordingly. If the patient needs new treatments without hospitalization, the ANA contacts the BRS to make these appointments. If the patient no longer needs treatment, he/she is released. Each time, the ANA registers the movement of the patient in his/her or her patients' file and contacts the BRS to inform 


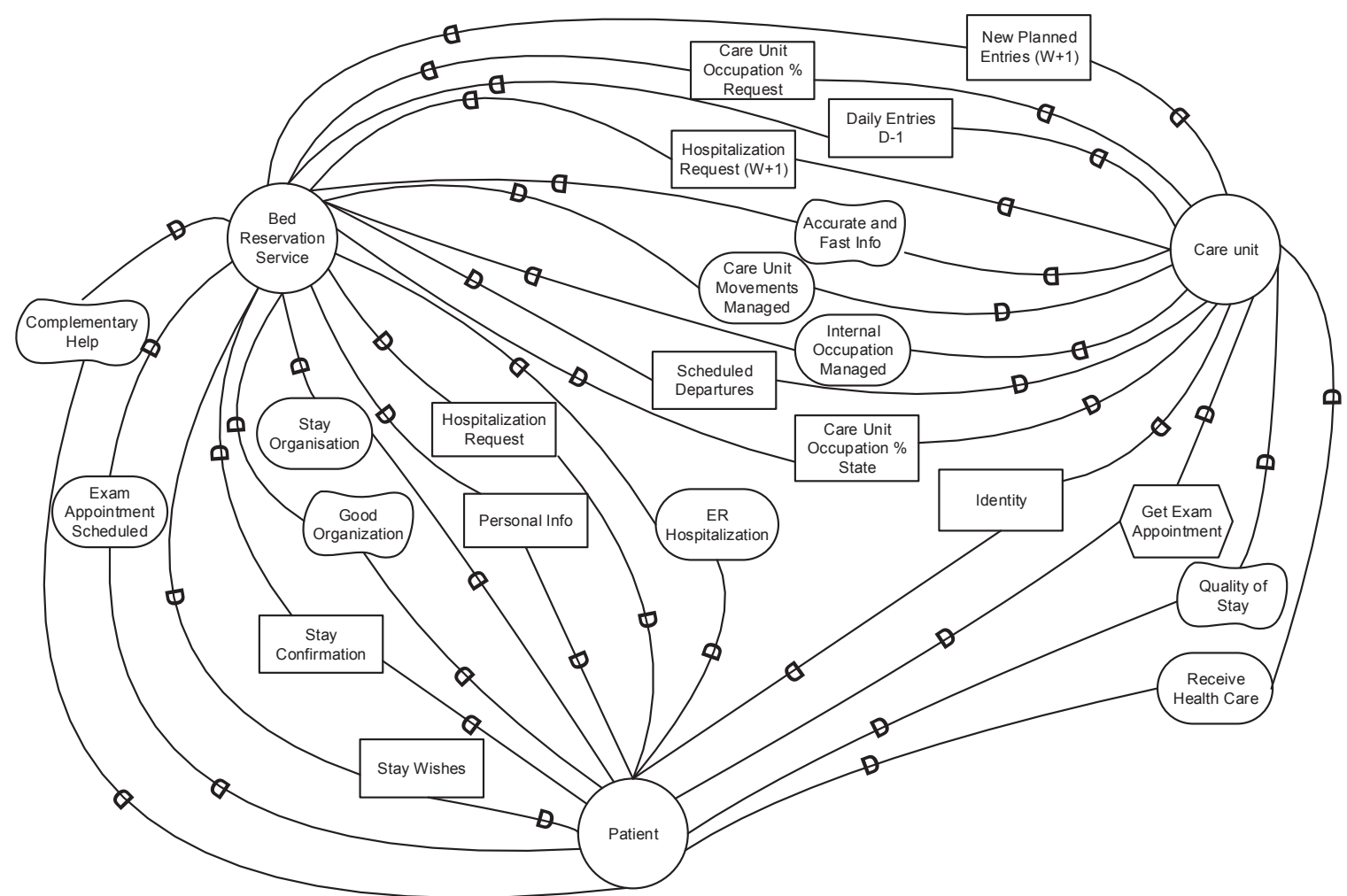

Fig. 6. Refining Strategic Dependencies for the Bed Reservation.

them of the extension of stays, moves or release of the patient. This way the BRS can have a better insight in the bed occupancy of each CU and make a better bed occupancy planning.

A broader requirements analysis for the Bed Reservation problem focuses incrementally on refining the strategic dependency model proposed above as depicted in Fig. 6. Three actors are involved: the Bed Reservation Service, the Care Unit and the Patient itself. The Bed Reservation Service and Care Unit are strongly interdependent of each other. The main goal dependencies Care Unit Movements Managed and Internal Occupation Managed point out that the processes of these actors are correlated through the fulfillment of these goals. The first one consists of planning and organizing patient entries and transfers between CUs. Without having reached this goal, the ANA will not be able to manage the CUs. Similarly, without internal management, the CUs could not transmit patient movements to the BRS, hence organizing efficiently emergency entries. Moreover, a double softgoal dependency connects both actors regarding the quality of the information exchanged and the impact on the adequate realization of their processes.

\section{Rationale analysis of the bed reservation process (AS-IS situation)}

Once this core first requirements analysis has been done, the strategic rationale model supplements the requirements analysis to express cognitive elements of the process and their underlying intentionality. It consists of an "exploration" of what is going on "inside" the strategic actors. Considering the model refined in Fig. 6, we focus here on the reasoning of the main professional organizational actor involved in the Bed Reservation problem, namely the Bed Reservation Service with respect to first the Patient in Fig. 7 and then the Care Unit in Fig. 9.

Fig. 7 specifies the organization of scheduled stays, i.e., the case when the patient goes directly to the Bed Reservation Service with a Hospitalization Request. The analysis focuses on the top-level task Scheduled Stay Organization, decomposes it through task decomposition in subgoals and subtaks. Dependencies from and to the Patient now connect directly adequate and precise rationale elements of the Bed Reservation Service. This cognitive decomposition will allow us later to explore alternatives.

Tasks are detailed using BPMN as process workflow as explained above. Due to a lack of space, we only detail in Fig. 8 the Inform task. Other BPMN workflows can be found in Wautelet (2017). The patient will be reminded by the BRS two weeks before his/her appointment to ensure the patient will show up at his/her appointment. Every day the BRS checks the appointment agendas and looks up the information needed for the notification document. This includes where and when the patient is expected, with whom he/she has an appointment and also the administrative data needed to address the notification document. Then they set up this notification document and send it to the patient by mail. There is also the possibility that the patient calls for a change of appointment or that the ANAs or doctors change their appointment. In that case they also inform the BRS of their change.

On the one hand, as seen, Fig. 7 and the BPMN workflows associated with it modeled the process of the bed reservation service 


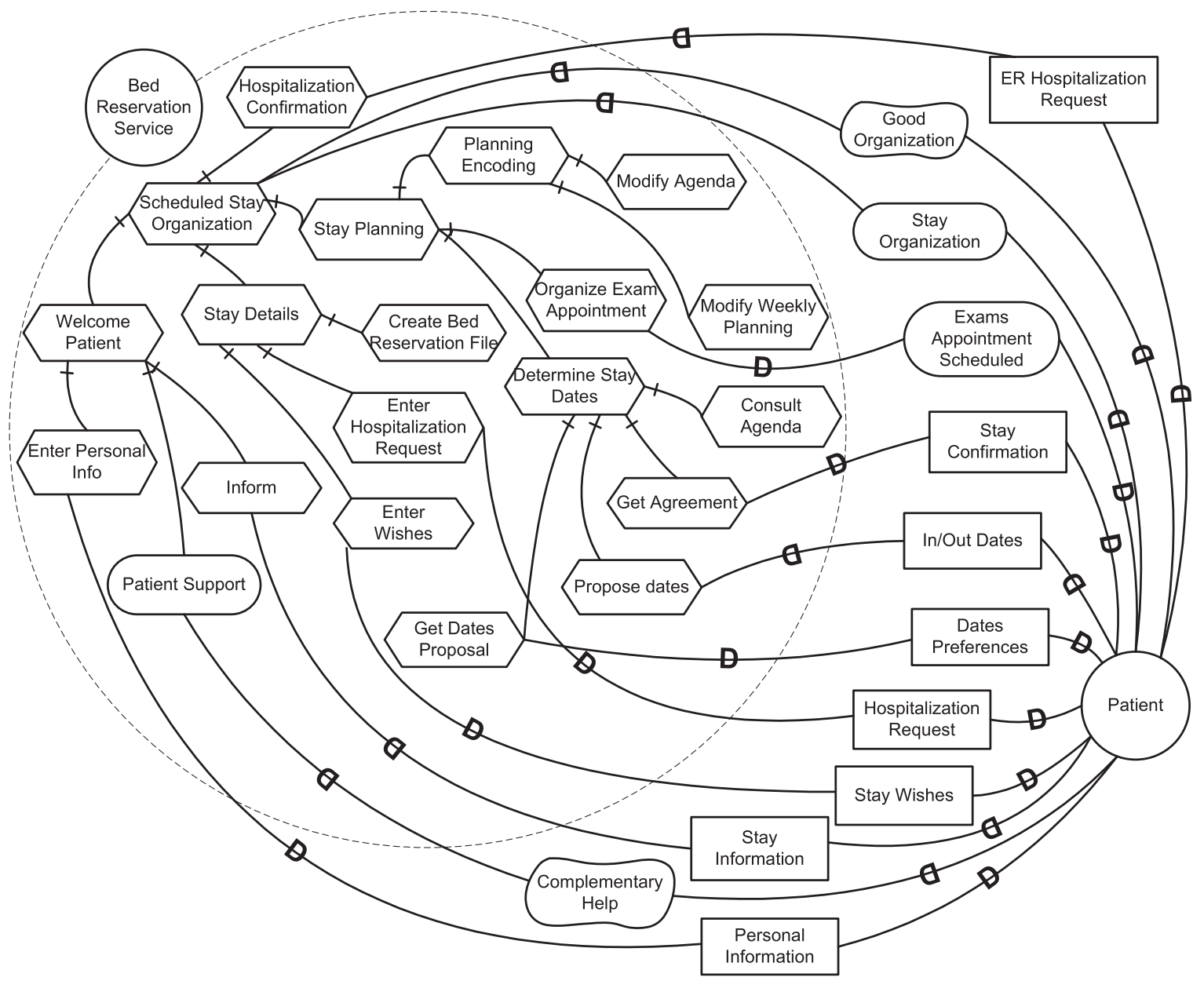

Fig. 7. The Bed Reservation Service Rationale.

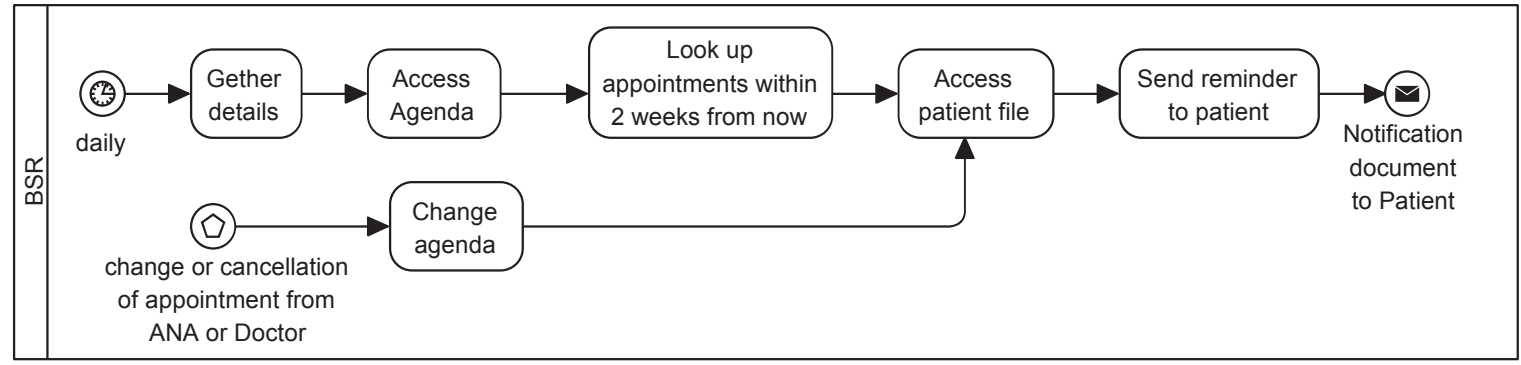

Fig. 8. The Inform Task Workflow.

with respect to the recording of a scheduled hospitalization request. On the other hand, Fig. 9 specifies the other activities of the bed management process: Organizing Non-Scheduled Stays, Care Unit Occupation Management and Patient Stay Management. Again, MeanEnds Analysis allow to point out alternatives such as the goal Answer to Requests for ER Hospitalization that can be accomplished either by Finding a Bed in the adequate unit or Finding a Temporary Bed in the another unit.

Task Care Unit Occupation Management (of Fig. 9) is detailed as a business process in Fig. 10: the CU checks the hospitalization agenda for the bed occupancy. They search for a bed slot and enter the data needed for the hospitalization and link the patients data to the chosen bed slot. If there is no available bed slot, an external transfer is needed. Other detailed workflows can be found in Wautelet (2017).

The next step is to use the strategic rationale to determine if an actor has the capability to accomplish functions, if the process is operationable and viable and if the beliefs are satisfied. Due to a lack of space, we only depict Fig. 11 that analyzes the reasoning and explores alternatives related to the Scheduled Stay Organization with respect to those four aspects mentioned just above. Make, Help, 


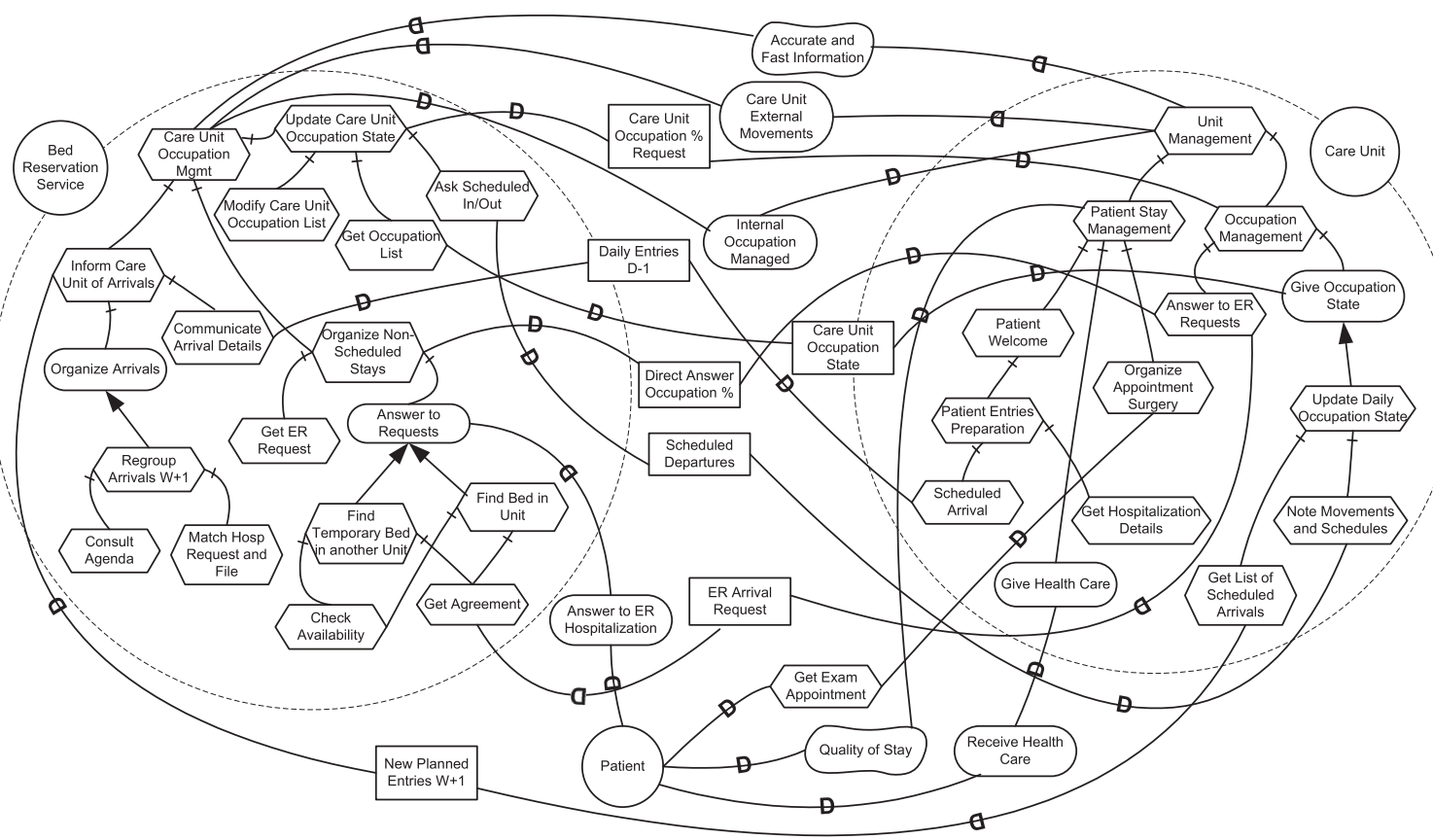

Fig. 9. The Care Units Rationale.

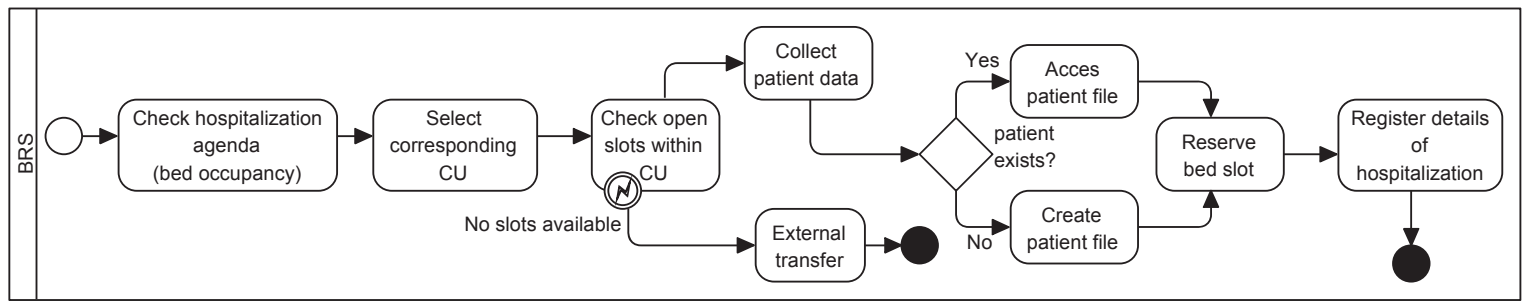

Fig. 10. The Occupation Management Workflow.

Break and Hurt links indicate respectively positive $(+)$, very positive $(++)$, negative $(-)$ and very negative $(--)$ goal contributions. These specifications help choose the adequate configuration with respect to trade-offs and compromises. For instance, the realization of the Let Administration take Appointments task hurts the fulfilment of the Synchronize Medical Administration while on the contrary Take Appointment helps it. This contribution analysis also helps to identify issues which could be helpful when designing the system architecture. Here it is clear that the use of office-based non-integrated procedures such as User Agenda and Copy Files really hurts architectural non-functional requirements such as Quality.

Again, tasks are detailed as processes using BPMN. For instance, the Organize Exam Appointment is depicted in Fig. 12. In this task, either a patient makes the appointment himself or the ANA of a CU calls the BRS to make an appointment for a hospitalized patient. The BRS then checks the appointments agendas (agendas for consultations and/or hospitalizations) to look for an open slot according to the demand. They also open the file of the medical staff needed for the treatment of that patient to see if they are available on the chosen moment. If everything is tuned to the best ability when planning the appointments manually, they register the data of the patient in the agenda and the appointment date in the patients' file. The BRS then gives the patient a confirmation for his/her appointment.

\section{Functions of the software system TO-BE}

Analysis of late requirements describes the (multi-agent) system-to-be (developed and adopted) within its operational environment along with relevant functions and qualities. It results in a specification which describes all functional and non-functional requirements for the system-to-be. The information system is represented as one or more actors which participate in a strategic dependency model, along with other actors from the system's operational environment. In other words, the system comes into the picture as one or more actors who contribute to the fulfillment of stakeholder goals. For our bed management problem, the Bed Management Planner is the introduced system represented as an actor in Fig. 13. Due to lack of space, the strategic dependency is directly combined here with strategic rationale models. Indeed, although a strategic dependency model provides hints about why processes are structured in a certain way, it does not sufficiently support the process of suggesting, exploring, and evaluating 


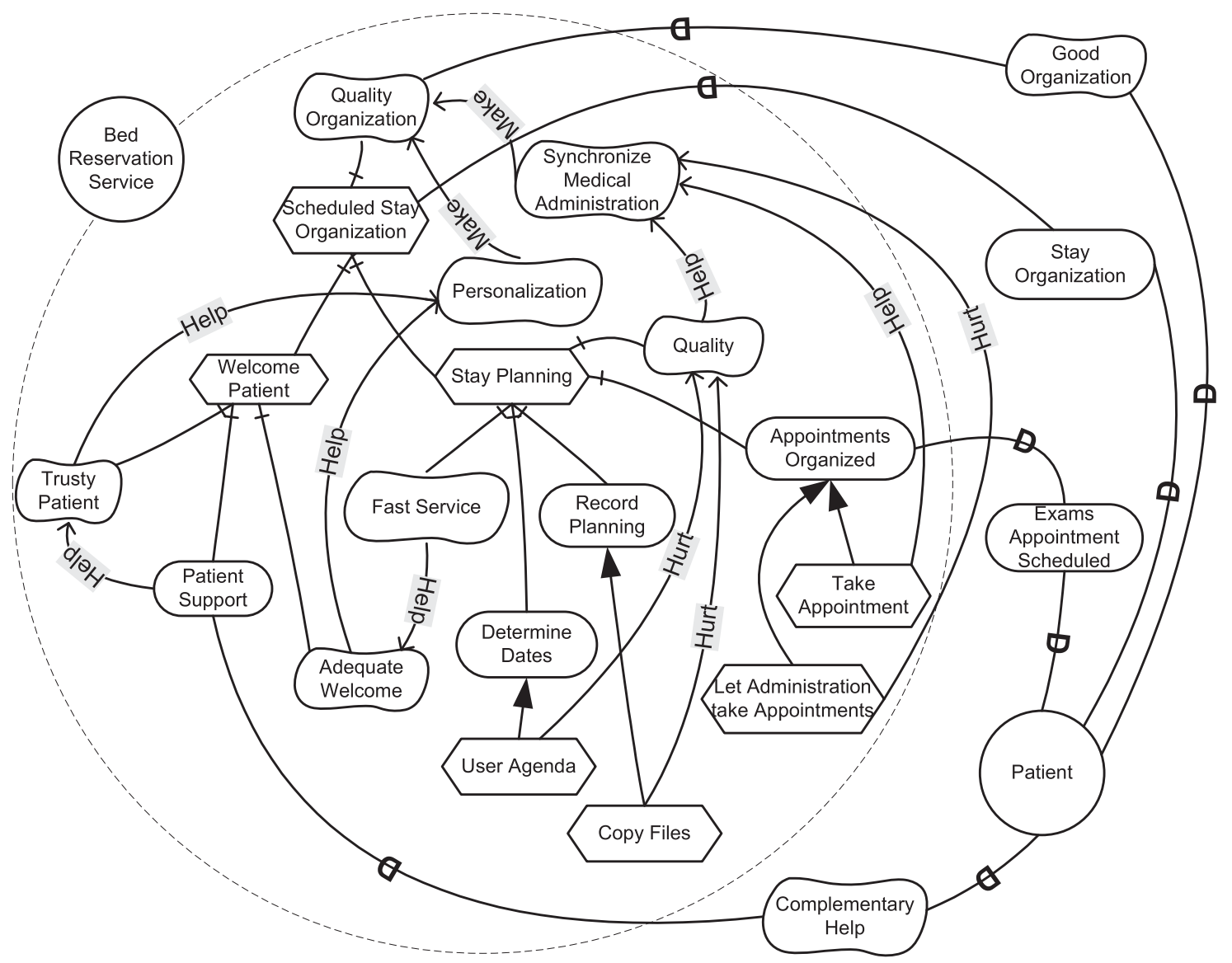

Fig. 11. Issues Identification through Rationale Modeling.

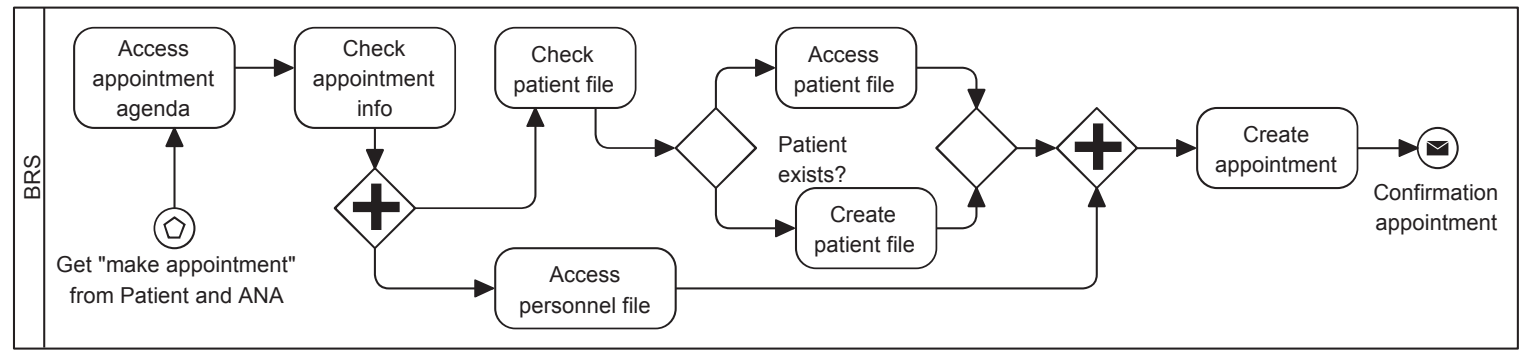

Fig. 12. Organizing Exam Appointment Process.

alternative solutions. As late requirements analysis proceeds, the Bed Management Planner is given additional responsibilities, and ends up as the depender of several dependencies. Moreover, the system is decomposed into several goals, tasks, resources, ... which take on some of these responsibilities. This decomposition and responsibility assignment is realized using the same kind of meansends analysis along with the strategic rationale analysis illustrated in Fig. 13.

Tasks to be implemented in the system-to-be are also modeled in details using workflows and other types of design diagrams such as those detailed in Section 9.2. For instance, for the sequence flow of the Organized Scheduled Arrival to be implemented by the Bed Management Planner System, the (system) process is described in Fig. 14. The registration enters the ID card in the card reader, so that the system can copy all relevant medical information from it. Although the patient file should already have been built in the preregistration process, the system checks this again, this to be sure the patient data is actually admitted into the system. The system then records the arrival of the patient in real-time and adds this data to the patient file. The next step is to check the validity of the badge of the patient. If the patient does not have a badge yet, the system automatically creates one now; else, the system just reads data from it. The system determines the type of the patient (see A) by reading his/her file. If the patient comes in for a Planned Hospitalization, the system checks his/her pre-admission data and informs the dietary service of his/her arrival. If the patient comes in for a consultation, these steps are not carried out. Afterwards, the patient is informed on the CU he/she has to go to. 


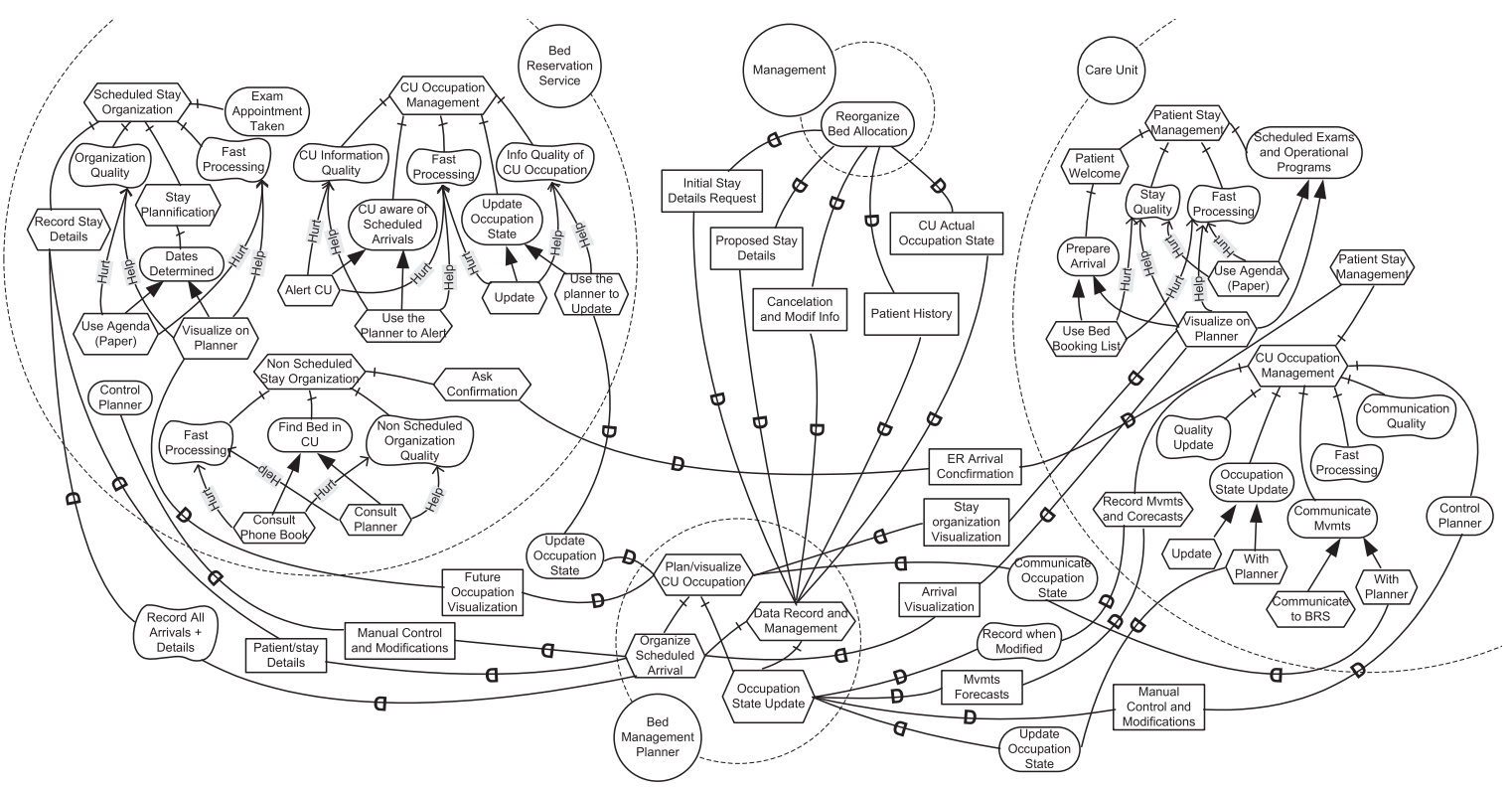

Fig. 13. Functions of the Software System TO-BE for the BRS and the CU.

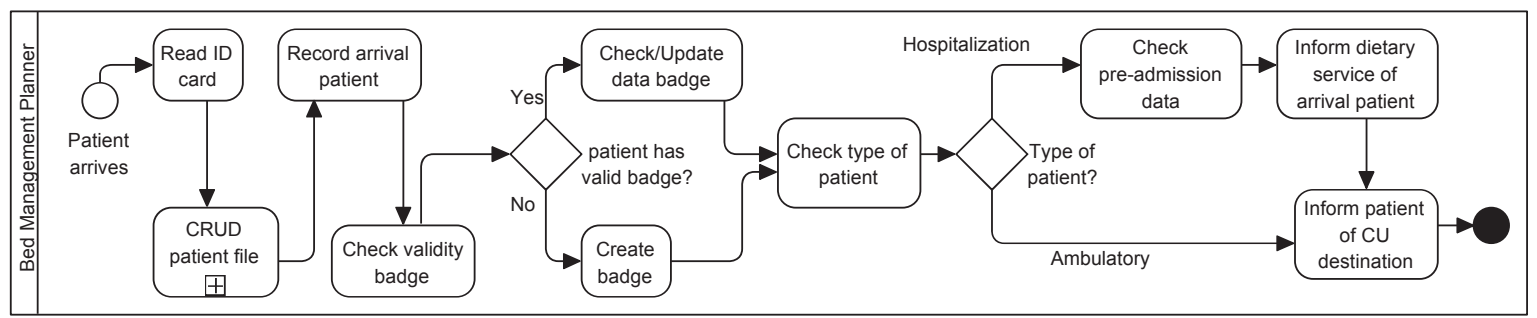

Fig. 14. BPMN process for the Organized Scheduled Arrival System Process.

\section{Towards a multi-agent software architecture}

The MAS architecture itself is depicted in Section 9.1 while Agent Detailed Design is overviewed in Section 9.2.

\subsection{Multi-agent architectural design as a joint-venture}

Fig. 15 suggests a possible assignment of system responsibilities, instantiating the (multi-agent) joint venture architectural style to guide the design of the system architecture. A system architecture constitutes a relatively small, intellectually manageable model of system structure, which describes how system components work together.

The selected MAS architectural joint venture style is a decentralized pattern based on service agreement between two or more principal software agents who benefit from operating at a larger scale and reuse the experience and knowledge of each other. The strategic operationalization and coordination of the joint venture style is delegated to a Joint Management agent, who coordinates tasks and manages the sharing of knowledge and resources.

Our system is thus decomposed into three principal agents (Bed Administration App, Accounting Processor and Patient Treatment Processor) controlling themselves on a local dimension and exchanging, providing and receiving services, data and resources with each other.

Each of them delegates authority to and is controlled and coordinated by the joint management agent (Bed Planner) managing the system on a global dimension. Bed Administration App interacts primarily with the Bed Reservation Service and Administrative Nurse Assistants which play the role of Bed Selector to provide them with a usable front-end desktop or mobile application. The Care Unit App keeps track of all information about patients, their movements, doctor visits, medico-technical exams, medicine received and other data of strategic and tactical importance for the management and decision making in the Hospital. Billing Processor is in charge of the secure management of invoices, and other financial data; also of interactions to Medical Insurance Organizations. Bed Planner manages all of them controlling security gaps, availability bottlenecks and adaptability issues. All four sub-agents need to communicate and collaborate in running the system. For instance, Bed Administration App communicates to Billing Processor relevant patient information required to process bills. The Care Unit App collects and structures all the data coming from the Bed Administration App and Billing 


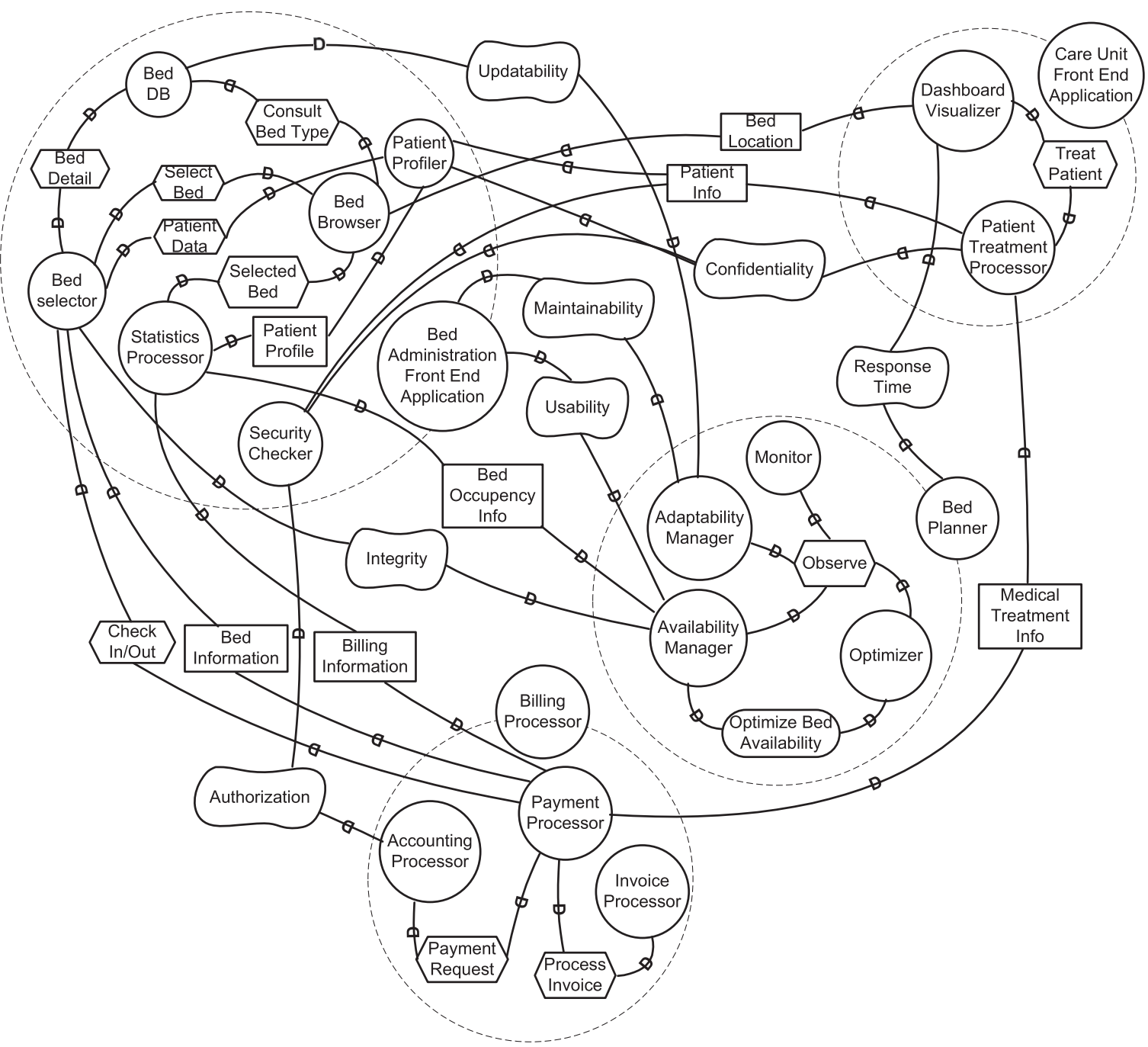

Fig. 15. The Bed Reservation Service System as a Joint Venture MAS Architecture.

Processor in order to produce statistical analysis, historical charts and other decision making information.

In the following, let us consider the further detailed information of the Bed Administration App. This agent is in charge of managing the entire stay of a hospitalized patient. We point out that the MAS intends to provide users like the Bed Reservation Service or the Administrative Nurse Assistants with various functions for bed selection and overview (per CU, per floor, per room, per patient, etc.).

Bed Administration App is also responsible for providing an overview of the bed occupancy in the Care Units. We assume that different Care Units using the MAS may want to provide patient with different levels of service with respect to their insurance, allergies, religion or simply willingness to pay for an upper level service (e.g., individual room, collective room, room with a view, tailored meals, television, internet access, ...)

Finally, Bed Administration App also initializes the kind of processing that will be done (by Billing Processor) for a given patient stay (payment done by insurance, payment done by patient). We assume that different stakeholders using the MAS may be processing various types of demands, such as those listed above differently and that patients may be requiring different kinds of treatments (doctor visits and medico-technical exams).

To accommodate the responsibilities of Bed Administration App, we introduce sub-agents: Bed Browser to manage beds presentation as a user friendly Graphical User Interface (GUI), Bed Selector to select and assign beds, Patient Profiler to track patient data and produce patient profiles, and Bed $D B$ to deal with the consistent record of the bed statuses.

Moreover, to cope with the identified software quality attributes (Security, Availability and Adaptability), Bed Administration App is further refined into four new system sub-agents Availability Manager, Security Checker and Adaptability Manager each of them assuming one of the main softgoals (and their more specific subgoals) and observed by a Monitor.

Billing Processor is decomposed into Payment Processor to aggregate the Bed Occupancy Information and deal with the billing details, Accounting Processor to interact with Medical Insurance Organizations (not represented in Fig. 15) and Invoice Processor to deal with invoice information. Finally, Care Unit App is refined into Dashboard Visualizer producing charts, reports, audits, sales, turnover 
forecast, and Patient Treatment Processor ensuring the data integration with third party medical software systems or (smart) medical devices used by doctors.

\subsection{Agent detailed design}

Detailed design is intended to introduce additional detail for each architectural component of a system. In our case, this includes agent communication and agent behavior. To support this phase, we propose to adopt existing agent communication languages like FIPA-ACL (F.F. for Intelligent Physical Agents, 2002; Poslad, 2007), message transportation mechanisms and other concepts and tools. One possibility is to adopt extensions to UML, like AUML, the Agent Unified Modeling Language (Guedes and Vicari, 2009) proposed by the FIPA (Foundation for Physical Intelligent Agents) (F.F. for Intelligent Physical Agents, 2002) and the OMG Agent Work group.

To illustrate the use of AUML, the rest of the section concentrates on the Patient Treatment Processor actor and the check out dependency. Our target implementation model is the Belief-Desire-Intension (BDI) model (Bratman, 1999; Rao et al., 1995; Casali et al., 2011; Pokahr et al., 2014; Wautelet and Kolp, 2016), an agent model whose main concepts are Beliefs, Desires and Intentions. As indicated in Fig. 19, we propose to implement $i^{*}$ tasks as BDI intentions (or plans).

To specify the checkout task, AUML allows us to use templates and packages to represent checkout as an object, but also in terms of sequence and collaborations diagrams.

Fig. 16a introduces the checkout interaction context which is triggered by the checkout Communication Act (CA) and ends with a returned information status. When the Patient wants to checkout, the Patient Treatment Processor asks the Payment Processor to process orders. In turn, the latter sends a payment request CA to Accounting Processor which informs him about the status (failure/success) of its internal processing. In case of success, Payment Processor concurrently asks Invoice Processor to process the invoice (and send a visualize detail CA to Dashboard Visualizer) and sends billing information to Statistics Processor.

This diagram only provides basic specification for an intra-agent order processing protocol. In particular, the diagram stipulates neither the procedure used by the Patient to produce the checkout CA, nor the procedure employed by the Patient Treatment Processor to respond to the CA.

As shown by Fig. 16b, such details can be provided by using levelling (Guedes and Vicari, 2009), i.e., by introducing additional interaction and other diagrams. Each additional level can expressinter-agent orintra-agent dialogues. At the lowest level, specification

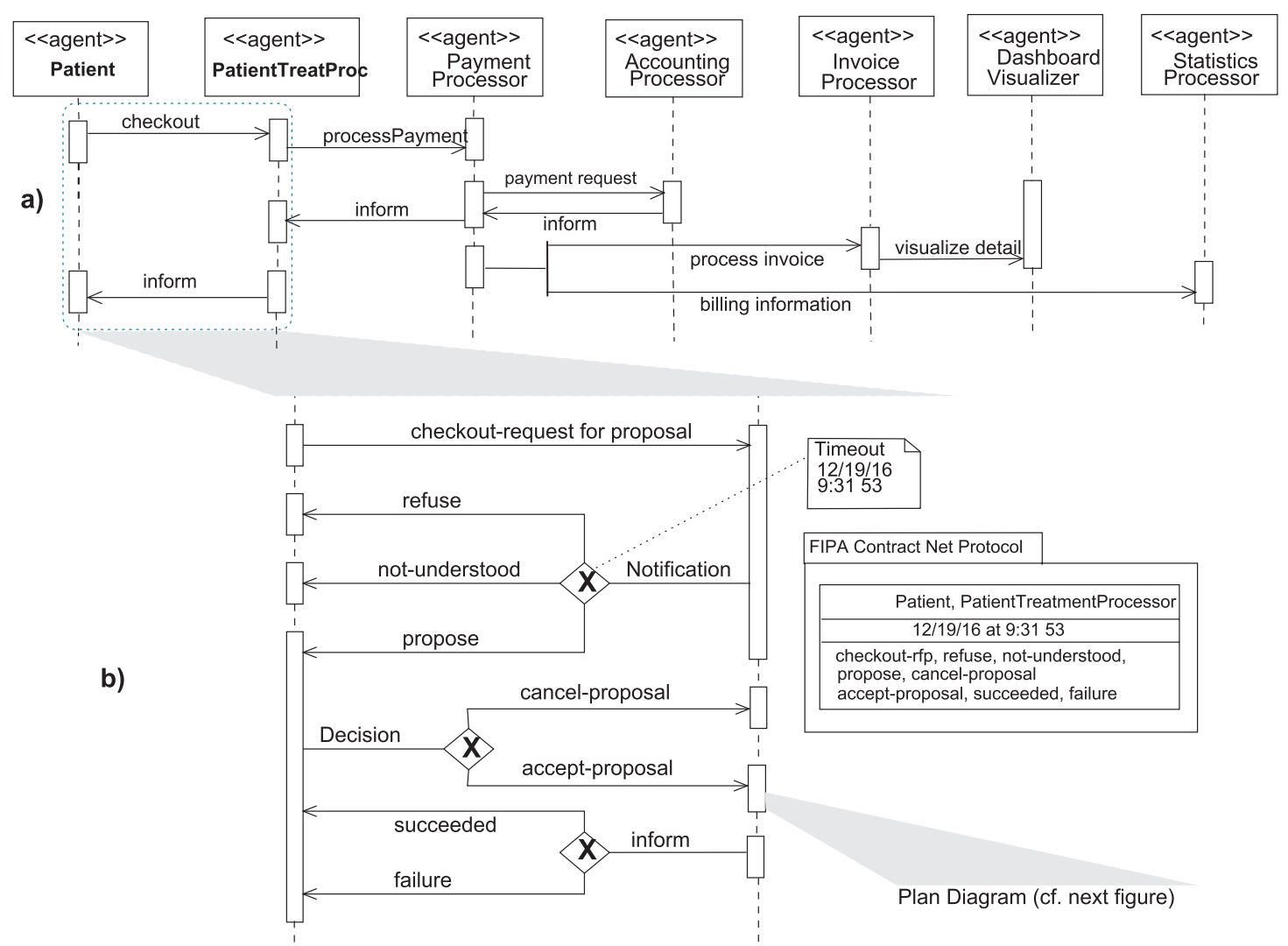

Fig. 16. Agent Sequence Diagram (a), and Agent Interaction Protocol (b) Focusing on a Checkout Dialogue. 


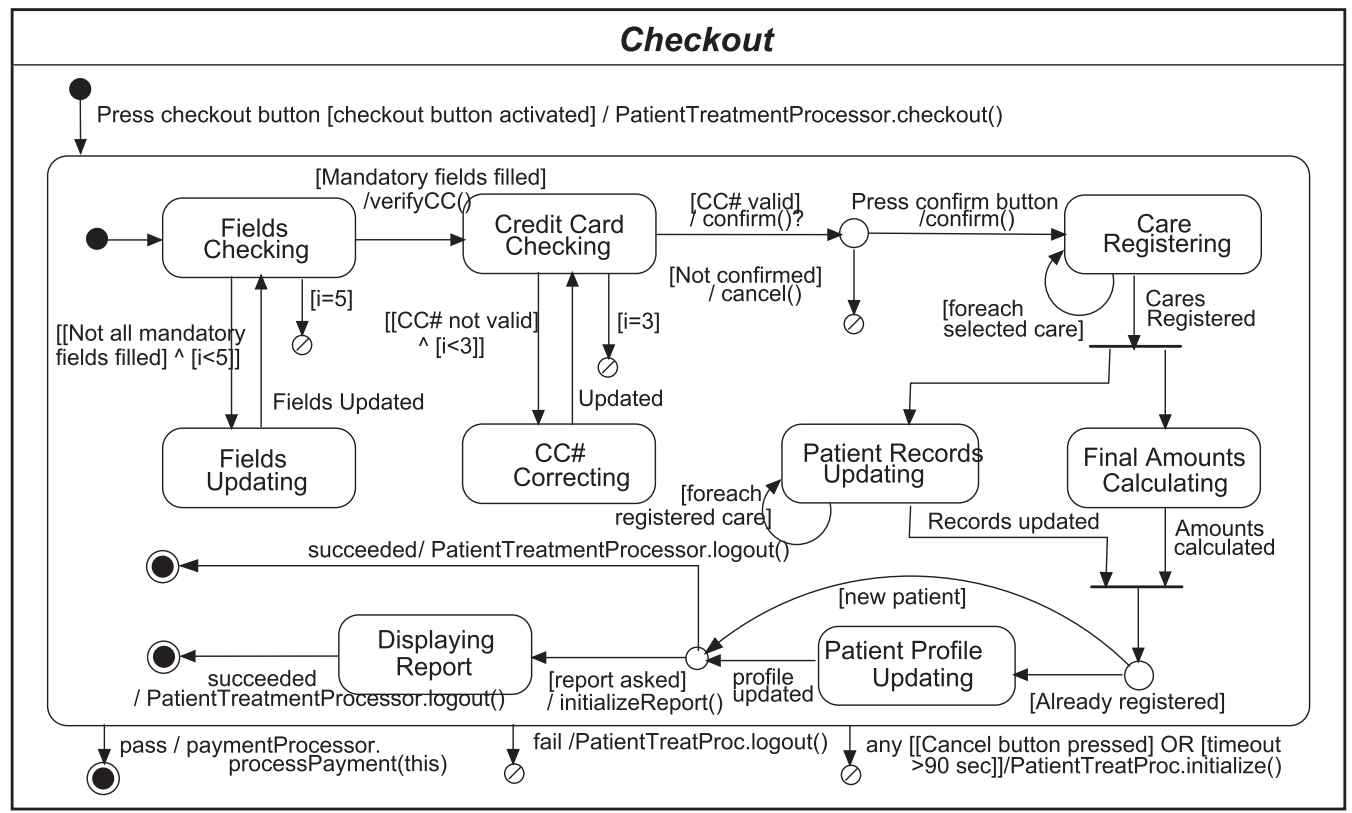

Fig. 17. A Plan Diagram for Checkout.

of an agent requires spelling out the detailed processing that takes place within the agent. Fig. 16b focuses on the protocol between Patient and Patient Treatment Processor which consists of a customization of the FIPA Contract Net protocol. Such a protocol describes a communication pattern among agents, as well as constraints on the contents of the messages they exchange. When a Patient wants to check out, a request-for-proposal message is sent to Patient Treatment Processor, which must respond before a given timeout (for network security and integrity reasons). The response may refuse to provide a proposal, submit a proposal, or express miscomprehension. The diamond symbol with an " $\times$ " indicates an "exclusive or" decision. If a proposal is offered, Patient has a choice of either accepting or canceling the proposal. The internal processing of Patient Treatment Processor's checkout plan is described in Fig. 17.

At the lowest level, we use plan diagrams (Castro et al., 2002), to specify the internal processing of atomic agents. Each identified plan is specified as a plan diagram, which is denoted by a rectangular box. The lower section, the plan graph, is a state transition diagram. However, plan graphs are not just descriptions of system behavior developed during design. Rather, they are directly executable prescriptions of how a BDI agent should behave (execute identified plans) to achieve a goal or respond to an event.

The initial transition of the plan diagram is labeled with an activation event (Press checkout button) and activation condition ([checkout button activated]) which determine when and in what context the plan should be activated. Transitions from a state automatically occur when exiting the state and no event is associated (e.g., when exiting Fields Checking) or when the associated event occurs (e.g., Press cancel button), provided in all cases that the associated condition is true (e.g., [Mandatory fields filled]). When the transition occurs any associated action is performed (e.g., verify CC()).

The elements of the plan graph are three types of node; start states, end states and internal states, and one type of directed edge; transitions. Start states are denoted by small filled circles. End states may be pass or fail states, denoted respectively by a small target or a small no entry sign. Internal states may be passive or active. Passive states have no substructure and are denoted by a small open circle. Active states have an associated activity and are denoted by rectangular boxes with rounded corners. An important feature of plan diagrams is their notion of failure. Failure can occur when an action upon a transition fails, when an explicit transition to a fail state occurs, or when the activity of an active state terminates in failure and no outgoing transition is enabled.

Fig. 17 depicts the plan diagram for checkout, triggered by pushing the checkout button. Mandatory fields are first checked. If any mandatory fields are not filled, an iteration allows to update them. For security reasons, the loop exits after 5 tries ([i $<5]$ ) and causes the plan to fail. Credit Card validity is then checked. Again for security reasons, when not valid, the CC\# can only be corrected 3 times. Otherwise, the plan terminates in failure. The patient is then asked to confirm the CC\# to allow item registration. If the CC\# is not confirmed, the plan fails. Otherwise, the plan continues: each care is iteratively registered, final amounts are calculated, patient records and profiles are updated and a report is displayed. When finally the whole plan succeeds, the Patient Treatment Processor automatically logs out and asks the Payment Processor to initialize the payment. When, for any reason, the plan fails, the Patient Treatment Processor automatically logs out. At anytime, if the cancel button is pressed, or the timeout is more than $90 \mathrm{~s}$ (e.g., due to a network bottleneck), the plan fails and the Patient Treatment Processor is reinitialized.

Dynamics specifications such as processes modeled by plan diagrams in Tropos can be formalized using ConGolog (De Giacomo et al., 2012). Primitive actions can be defined in terms of pre- and post-conditions and decomposed into procedures using modeling constructs like sequencing $\left(a_{1} ; a_{2}\right)$, conditional (if-then), iteration (while $<$ condition $>d o$ ), concurrent activities $\left(a_{1} \| a_{2}\right)$, priority 


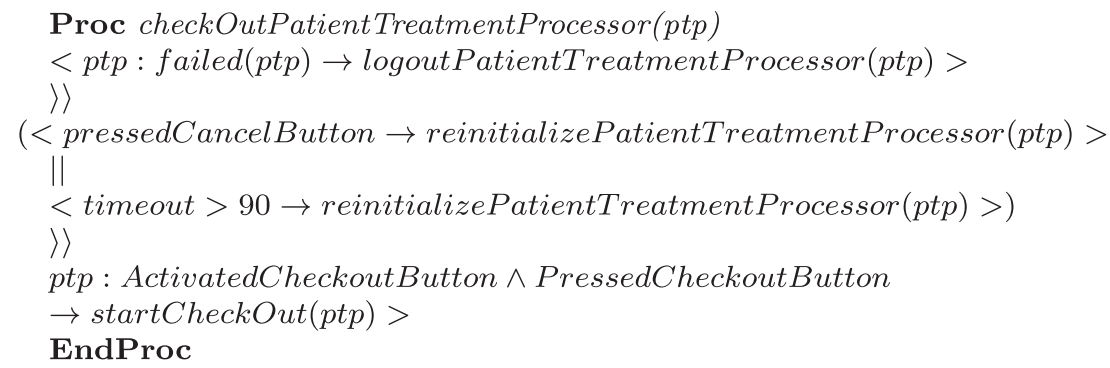

Fig. 18. ConGolog-like specification for the checkout plan from Fig. 17.

$\left.\left.\left(a_{1}\right\rangle\right\rangle a_{2}\right)$, non-deterministic choice $\left(a_{1} \mid a_{2}\right)$, interrupt $(<x: \phi \quad \rightarrow \quad \sigma>$ where $x$ is a list of variables, $\phi$ a trigger condition and $\sigma$ a body), ...In addition to offering programming language-like structures for describing processes ConGolog underlying logic is designed to support reasoning with respect to process specifications and simulations. Fig. 18 gives some ConGolog specifications for the checkout plan graph of Fig. 17.

\section{Agent-oriented implementation}

The Jadex agent platform and the angular 2.0 framework (Darwin and Kozlowski, 2013) have been used for implementing respectively the MAS and the graphical user interface to interact with the agents. The latter allows developing and access applications on multiple platforms (i.e. a browser and a mobile device such as mobile phone or a tablet). Jadex was originally developed as an add-on to JADE (Java Agent DEvelopment Framework) (Bellifemine et al., 2007) in order to provide the latter with a reasoning layer to support the BDI model.

Jadex agents can be considered autonomous software components that have explicit goals to achieve or events to cope with (i.e. the desires). To describe how they should behave in order to achieve these desires, agents are programmed with a set of plans (intentions). Each plan describes how to achieve a goal under different circumstances. Set to work, the agent pursues its given goals (desires), adopting the appropriate plans (intentions) according to its current set of data (beliefs) about the state of the world. A Jadex agent is composed of beliefs, goals, plans and capabilities. The latter is a concept used for modularization and reuse in agent (Braubach et al., 2005); the capability is composed of a set of plans, beliefs, and goals.

Fig. 19 summarizes the mapping from $i^{*}$ concepts to Jadex constructs and how each concept is related to the others within the same model. $i^{*}$ actors, (informational/data) resources, softgoals, goals and tasks are respectively mapped into BDI agents, beliefs,

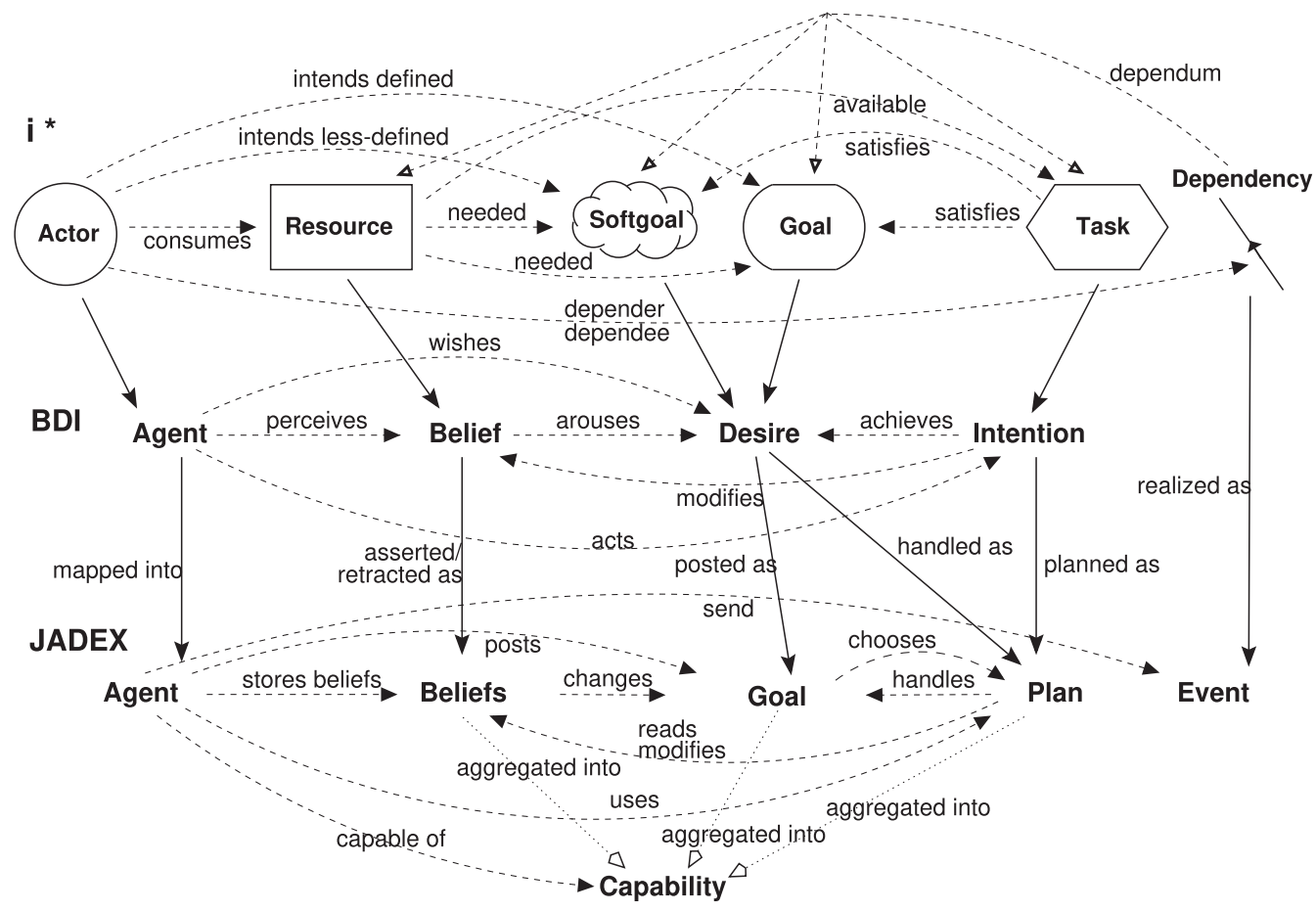

Fig. 19. $i^{*} / \mathrm{BDI} / \mathrm{JADEX}$ Mapping Overview. 


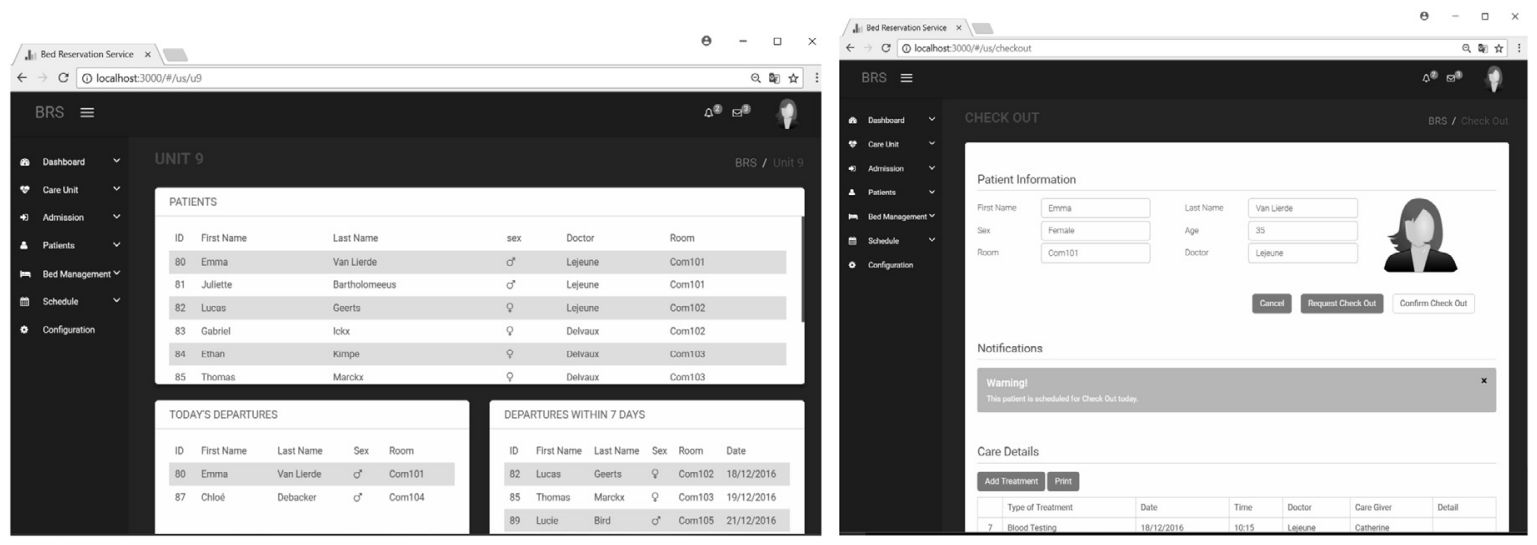

Fig. 20. Care Unit User Interface.

desires and intentions. In turn, a BDI agent will be mapped as a JADEX agent, a belief will be asserted (or retracted) as a database relation, a desire will be posted (sent internally) as a Goal (representing an objective that an agent wishes to achieve), and handled as a plan and an intention will be implemented as a plan. Finally, an $i^{*}$ dependency will be directly realized as an Event (received by agents from other agents).

In addition, the latest version of Jadex (numbered $3.0^{3}$ ), provides the Representational State Transfer (REST) Web Service directly within the platform which facilitates our implementation since we need to provide a web-based and a mobile interface. We also use JSON formats for exchanging data between agents and the end-user interface; Angular being fundamentally based on JSON data.

Figs. 20 and 21 depict respectively the implemented user interface for managing the care units and some dashboards. The latter allows the caregivers to see the list of actual patients in their unit with the prediction of checkout patients for seven days. It also allows caregivers to see a checklist of care for each selected patient. The dashboard provides real-time state of bed availability for each service and the summary of admission type of the hospital.

\section{Validation of the MAS-based platform's support on the hospital strategy}

To validate our proposal, we have first proceeded to a modeling of the hospital global strategy; as evoked in the research method, this has been established through interviews. Then, in a second phase, we have crossed the main goals of this long term strategy in order to evaluate the impact of the MAS-based platform onto it. These elements are modeled using the NFR framework in Fig. 22.

The strategy of the hospital is concentrated around two main objectives, (i) Break even on medical reimbursements and (ii) Provide high quality care services. We only concentrate here on the presentation of the strategic objectives that are impacted by the MAS development (this with respect to the architectural scenario provided in Section 9.1).

As suggested in the first objective Break even on medical reimbursements, the long term financial viability of the hospital is from primary importance. This is further decomposed in the following sub-objectives:

- Minimize payment time of medical insurance organizations and patients. Financial management includes a timed balance between cost and income. If the payment delays of treatments by Medical Insurance Organizations and Patients can be lowered, payments that the hospital needs to fulfill are ensured and substantial amounts of money can be spared. That is why the Billing Processor that allows to automatically assign the treatments and other services to a specific patient and manage the invoicing has a (very) positive impact on this objective;

- Support in time access to rooms, medical acts and medico-technical exams. Extensive waiting periods for patients to get access to their rooms, medical acts and medico-technical exams induces global planning delays and other issues which leads to patient loss and has also a negative impact on the image. The Care Unit App with its business intelligence functions (dashboards and other analysis) allows to identify bottlenecks in patient flows which as a (very) positive impact on this objective. Similarly, the Bed Planner optimizes the bed occupation with a direct impact on waiting times, it has thus also a (very) positive impact on this objective;

- Develop acute care service. Acute care services imply active but short-term treatment for a severe injury or episode of illness, an urgent medical condition, or during recovery from surgery. Such services induce a minimal time for the Patient to remain in the hospital. The Care Unit App furnishing treatment flow optimization has a positive impact on this objective. The Bed Planner that allows to furnish real-time and short-term solutions for acute patients also has a positive impact on this objective. Finally, the Bed Administration App facilitates the assignment of beds in real-time for short periods and has thus a positive impact on the objective;

As suggested in the second objective Provide high quality care services, the quality of medical care services need to be of best possible quality. This is further decomposed in the following sub-objectives:

\footnotetext{
${ }^{3}$ see https://www.activecomponents.org.
} 


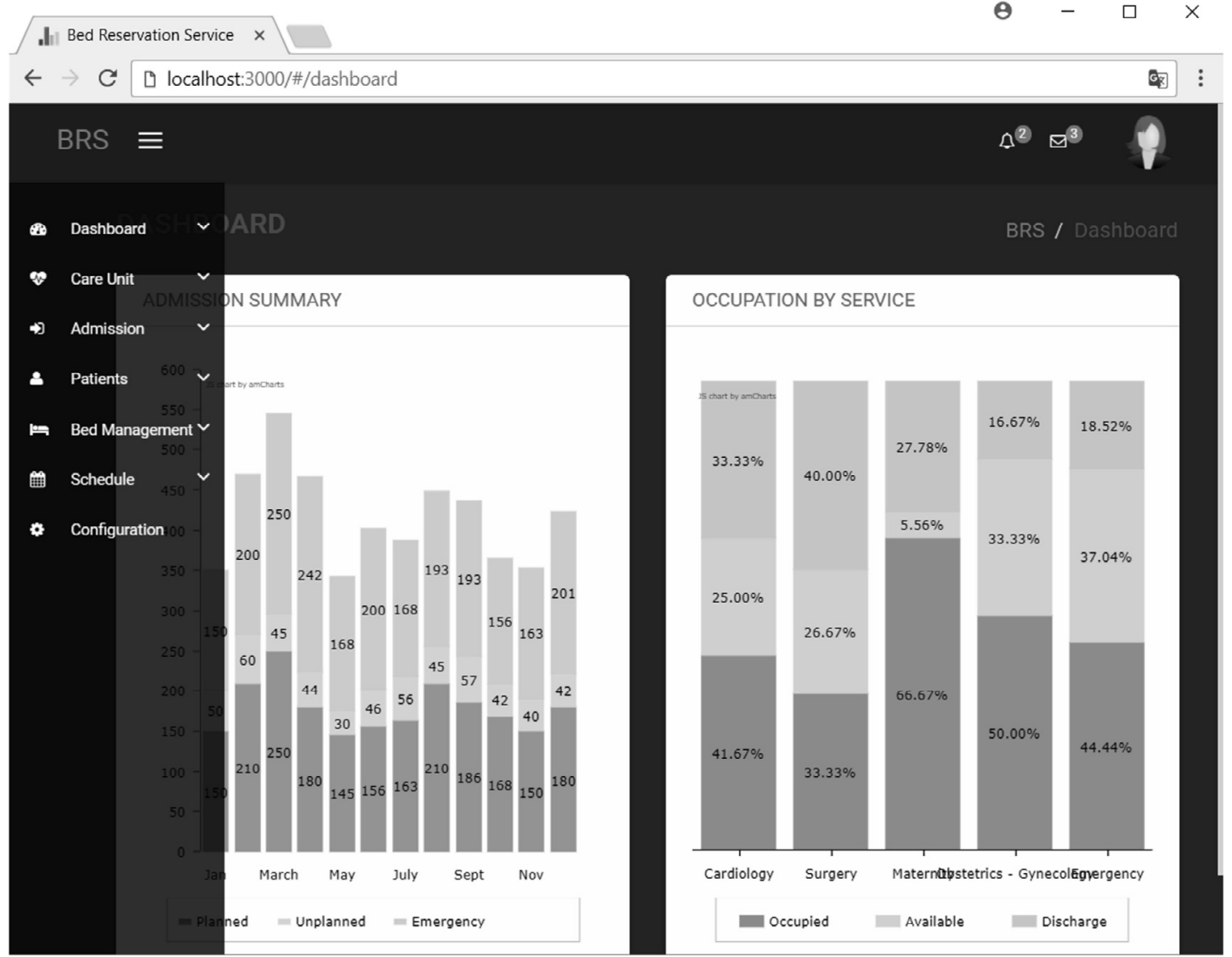

Fig. 21. Dashboard User Interface.

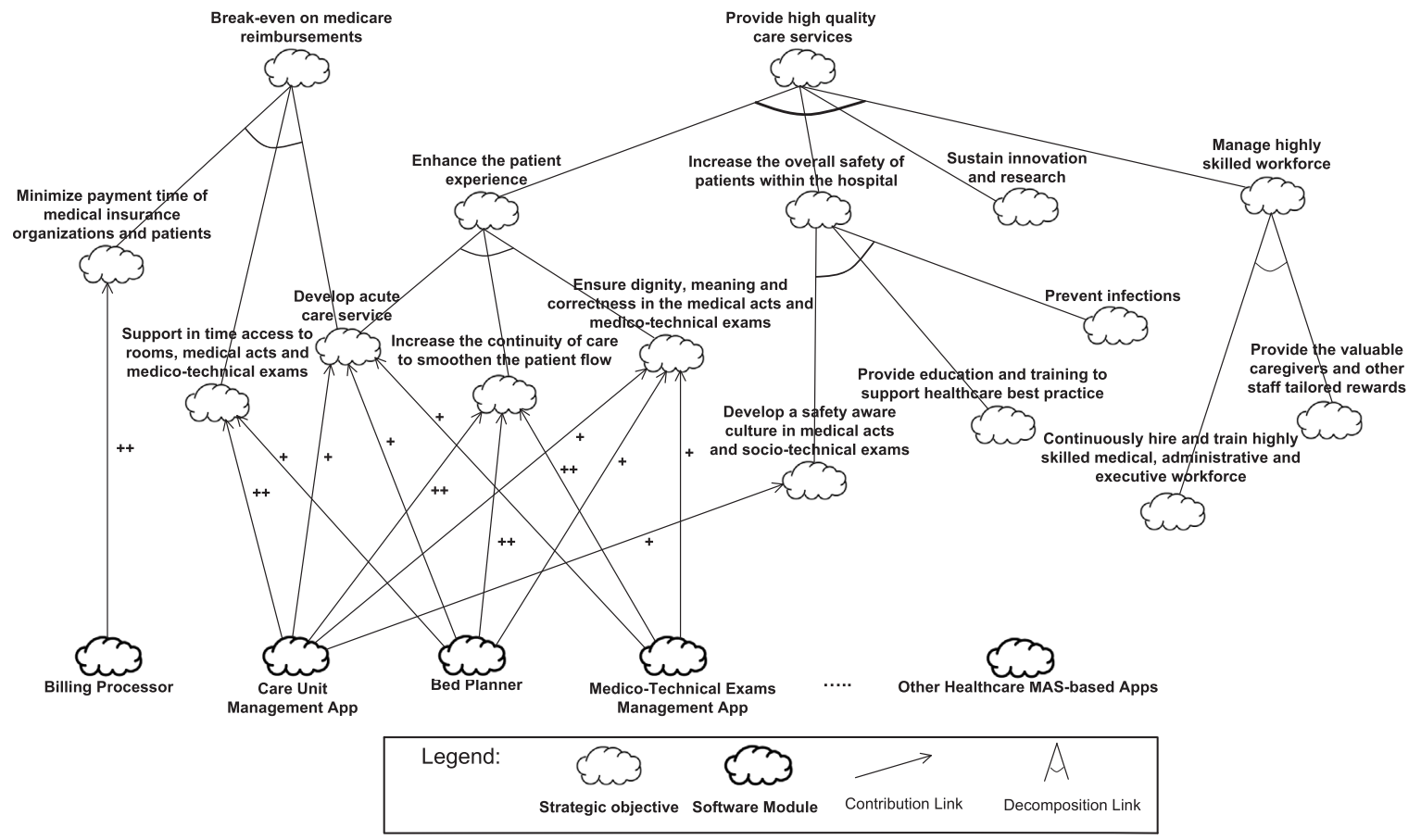

Fig. 22. Hospital's Strategic Objectives. 
- Enhance the patient experience is further decomposed in the following sub-objectives; we mention here:

-Develop acute care service. This objective has been explained previously; we point it out here because it constitutes our objective for enhances patent experience in the long-run;

-Increase the continuity of care to smoothen the patient flow. When the patient needs to get care and medico-technical exams, this can follow each other smoothly which leaves clear and long spots to rest or these can be at random moments in time which induces to be often disturbed. The Care Unit App with its dashboards and other analytical tools allows to identify bottlenecks in this process; it has thus a (very) positive impact on this objective. Similarly, the Planning App allows to take such constraints into account when planning the bed occupation; it has thus a (very) positive impact on this objective. Finally, the Bed Administration App allows to make real time changes in planning to take into account new constraints appearing on a custom basis; it has thus a (very) positive impact on this objective;

-Ensure dignity, meaning and correctness in the medical acts and medico-technical exams. The Care Unit App allows to query data about the continuity of care for hospitalized patients which allows to avoid overlapping and unnecessary care; it has thus a (very) positive impact on this objective. The Bed Planner allows to furnish beds to patients in a more optimal way avoiding waiting delays; it has thus a (very) positive impact on this objective. Finally, as for the previous objective, the Bed Administration App allows to make real time changes in planning to take into account new constraints appearing on a custom basis; it has thus a positive impact on this objective.

- Increase the overall safety of patients within the hospital can be decomposed in several sub-objectives; we only mention here: -Develop a safety aware culture in medical acts and socio-technical exams. Safety has been and still remains a key element to provide high quality care. The Care Unit App with its dashboards potentially pointing out issues in the patient-processes safety has a positive impact on this objective.

We can thus see that the Bed Planner module is aligned with various of the long term strategic objectives of the hospital. This is overviewed in the conclusion but with another perspective made of three areas: economic, social and cultural.

\section{Discussion}

\subsection{Impact of the research on the patient-centric approach}

As initially evoked in the paper, the core of our research relies in the patient-centric modeling of the hospital bed management processes. To such an end, we have used of a socio-technical approach leading to the development of a MAS-based software system with a run-time behavior at best aligned with the real life organization but also aligned with the strategic objectives. Such an approach leads to a software system that implicitly takes the wellbeing of each patient into account through ensuring their flow into the hospital being as smooth as possible.

Achieving global patient centricity in the hospital is nevertheless a more global task and requires an evolution not only of technology but also taking into account the best interest of the patient during each step of the medical processes and/or cares. Moreover, the hospital is subject to constant environmental evolution due to new care techniques, medical technologies, legal context, etc. By several aspects, the adoption of the MAS-based platform nevertheless constitutes more than a first step for a global patient-centric approach; it indeed:

- allowed the modeling (representation) of the reality a patient is going through and that is not necessarily well understood by deciders or technology professionals. This way, each step of the process but also each dependency could be identified and modeled. The set of models constitute a powerful tool that can be used to increase the global patient-centric approach;

- furnishes a platform able to flexibly evolve to include new technological or methodological progress related to the patient wellbeing;

- furnishes all kinds of data related to the patient hospital stay useful for decision making in the domain of patient wellbeing or any other domain.

Let us finally note that the platform has been developed in the context of the processes of a well-defined organization and that even if other hospitals have comparable processes, it cannot be adopted as such by other organizations. Some customization of the platform and/or process reengineering would in any case be necessary.

\subsection{SWOT analysis of the MAS-development project}

This section summarizes the Strengths, Weaknesses, Opportunities and Threats (SWOT) analysis performed for the entire research project.

\section{Strengths}

- Socio-technical approach to understand the dependencies between stakeholders and IT technologies;

- Alignment and support of the organization's strategic objectives;

- Base for a global approach of patient-centric healthcare;

- Open MAS for an easy adoption of newly introduced connected objects and smart devices; 
- Single source of patient-related data for analytics.

\section{Weaknesses}

- The MAS-based system is designed for a specific organization and has not been thought for a generic adoption in other hospitals.

\section{Opportunities}

- Trend for pervasive healthcare in the medical sector.

\section{Threats}

- Technology standardization in the medical sector pushed by a major ERP vendor.

\section{Conclusion}

The need for an optimal use of available resources induced by a huge demand for cares and medico-technical exams has lead to the use of innovative agent-based technologies to support the processes of a Belgian hospital. This allows to manage the daily operations but also to collect relevant data and information on these processes useful for decision making. This paper has studied the existing patient processes which lead to the identification of the issues related to bed management and the development of a MAS solution to support the various aspects of this complex problem. Indeed, the MAS behaves at run-time in line with the working processes of the hospital and furnishes relevant solutions to various kinds of stakeholders.

The use of distributed agent-based technologies for process support and decision making has a clear positive impact on the long term strategy of the hospital. Within this positive impact, we highlight below:

- economic benefits involve gains of time and money through process reengineering and optimization. We highlight Minimize payment time of medical insurance organizations and patients allowing a more profitable financial management; Support in time access to rooms, medical acts and medico-technical exam allowing a leaner approach so to reduce waste due to the non-use of resources and Develop acute care service by nature more cost-efficient than traditional patient stays;

- social benefits increase the wellbeing of hospital's patients. We highlight Increase the continuity of care to smoothen the patient flow to optimize balance of time devoted to care and rest. Ensure dignity, meaning and correctness in the medical acts and medico-technical exams to treat patients in a more consistent and respectful way;

- cultural benefits change the medical staff habits to furnish higher quality care services. Develop a safety aware culture in medical acts and socio-technical exams allows to higher the general safety level in medical acts.

The paper has mainly focused on the bed management aspects but other areas of the patient processes are or will be supported by the MAS solution in the future. The platform has been developed for the specific purpose of the evoked case, we did not take the approach of a software product line to build a solution that could then be tailored to other hospital or healthcare institutions. This is left for future work.

Future work also includes the complete development of a turn key business intelligence solution to better exploit the collected data. Even if, as seen in the paper, the solution is already available, bringing it to a higher level of automation through a graphical user interface furnishing in real time an answer to the most often used queries will ease its use, increase its adoption by the medical staff and deliver even more important benefits. Also, just partial exploitation of the available data is done so far, more work is required by data scientists to use it at its full potential.

\section{Appendix A. Hospital stakeholders}

\section{A.1. The ptient}

The patient is an individual receiving or registered to receive medical treatment. This role is crucial since the hospital main purpose is to provide care to it. The patient is nevertheless not a key user of the hospital support software system but has to be strictly followed-up during its hospital stay in order to provide him qualitative services. Multiple scenarios are possible for the patient. The first distinction to make is the nature of his/her admission into the hospital: either in emergency, or in non-emergency.

Within the non-emergency condition, a patient can come to the hospital for 3 reasons: either he/she comes to get an appointment, or he/she comes for a consultation (called ambulatory patient in the scenarios described in Section B), or he/she comes for a planned hospitalization. Each patient owns a "personal file" updated by the different services of the hospital.

\section{A.2. Care units}

The Care Units (CU) are the departments of the hospital. Physically, a CU is made of a set of (bed) rooms where patients reside in order to get services around a specialty (for example oncology, pediatrics, stomatology, reanimation, etc.). The emergency department 
(ED or emergency room (ER)) is a special CU devoted to the patients that require immediate care; the Emergency CU indeed manages all the non-scheduled patients entering the hospital. It notably requires to do simultaneously: analyze the patient's condition, look for or create his/her personal file and redirect the patient to the next most adequate place (either another CU, or a different place within the CU he/she is already in), in function of the patient's condition. The staff members of the Emergency Room (ER) look by themselves for an available slot in other CU's make the reservations for medico-technical treatments or consultations for a patient that needs to be transferred. This CU only looks for the availabilities on a very short term so that it is not in "competition" with the Bed Reservation Service.

\section{A.3. Healthcare team}

The Healthcare Team provides the patients with medical care. The latter is mainly composed of nurses and doctors also owning an individual file encompassing their personal data as well as, for doctors, the medical treatments they perform. A doctor is independent of a CU unless he/she is service head. Most of the nurses belong to a specific CU but, next to this, a set of "flying nurses" that do not belong to a particular CU exist. Another distinction must be made between the "flying nurses" operating in the daily shifts and the ones operating in the night shifts. Indeed, the former are allocated in function of the load of the different CU; this means that in the morning each CU enters its requirements for flying nurses for the present day into the IT system. Then, the nurses are allocated for the day to the most requiring CU (so according to their work load). For the night shift, the "flying nurses" simply walk from one CU to the other and work where required on a cases by case basis.

\section{A.3.1. Admission}

The admission is made of a set of desks where the patients need to register when they enter the hospital for an ambulatory consultation or a planned hospitalization. This department aims to record (in real time) the arrival of the patient in the database, of updating its personal data and to inform him on the destination he/she has to go. Nevertheless, the admission never makes appointments for a patient and does not manage non-planned entries. This is done by the BRS (see next Section).

\section{A.3.2. Bed reservation service}

The third administrative department that directly deals with the patient is the Bed Reservation Service (BRS). The BRS is a service that directly organizes all the aspects of the stay of a hospitalized patient (bed reservation, booking of medico-technical treatments and consultations, etc.).

The BRS must not be mixed with the work of the ANAs. The ANAs are working within a particular CU while the BRS works for the entire hospital as they are in charge of concretely managing the bed allocation to the patients. The ANAs do not belong to the BRS but they can be seen as the exclusive point of contact with (or representative of) the BRS within a CU (see further).

\section{Appendix B. Hospital patient-centric processes}

\section{B.1. Process of an ambulatory patient}

An ambulatory patient is a patient that comes to the hospital for a planned consultation; in other words, it is not an emergency situation and he/she does not benefit of a bed reservation in the hospital.

The journey of an ambulatory patient starts with setting an appointment. The appointment can be fixed by phone or in person at the BRS. The data relative to the appointment are registered whether the patient is already preexisting in the system or not.

When the patient arrives at the hospital for its consultation, he/she registers at the admission service. The patient can either be a new patient and his/her personal data must then be registered, or his/her data can already exist in the system. Each patient must be in possession of a badge (in the shape of a credit card) that indicates his/her name, sex, birth date and administrative number. This badge is printed on the healthcare (or examination) worksheets ${ }^{4}$ that the patient gets during his/her journey. The administrative number is unique and allows accessing the personal data of the patient and his/her health insurance. The badge is valid for a limited period of maximum one year and is checked by the admission at each patient entry; this allows updating the data or creating a new badge if the patient does not own one or if it is outdated. If he/she does not own a valid badge, the employee of the administration creates one and/or updates a series of administrative data by reading the personal identity card (this is an individual card owned by each Belgian citizen that notably integrates information on the its healthcare insurance). If the patient does not own such a card or for the information that missing, a manual registration is necessary (contact information, name of the healthcare insurance, etc.).

Once in possession of a valid badge, the patient can consult a doctor of the hospital if he/she has arranged a consultation. The doctor then indicates the delivered cares on the healthcare worksheet. The patient that comes for a consultation shall indeed be assigned a healthcare worksheet (that is logically linked with his/her badge). As evoked, this worksheet summarizes all the medical treatments performed during the consultation. At the end of the patient visit in the hospital, this healthcare worksheet is collected by the financial service that registers its content manually in the system for invoicing purposes of the services.

If the patient does not have a consultation or after a consultation, the patient may be required to have $\mathrm{x}$-rays and then goes to the

\footnotetext{
${ }^{4}$ Typically, the patient collects a set of these worksheets during his/her stay at the hospital, at each consultation he/she gets a new one, at each medico-technical exam or treatment another, etc.
} 
$\mathrm{X}$-ray service. In this service, the patient has one or more $\mathrm{x}$-rays. The medical treatments are here directly encoded in the information system by the radiologist in order to be automatically sent to the financial service for invoicing purposes.

Similarly, a patient can then have to (or not to) provide diverse samples for laboratory tests. In that case, he/she goes to the laboratory where the required samples are collected and analyzed. The medical treatments performed in the laboratory are directly registered in the system by the laboratory technician and automatically sent to the financial service in order to be invoiced to the patient.

The visit of the patient is then finished.

\section{B.2. Process of a hospitalized patient}

A hospitalized patient is a patient that presents himself for a planned hospitalization. In other words, it is not a situation that is relevant for the emergency CU and he/she benefits of a bed reservation at the hospital for a defined term.

Most of the time, in most of the cases, every patient that will be hospitalized must previously go through the BRS with its medical hospitalization demand to book the stay dates, the type of bed booked, etc.

The day of his/her entry, the patient goes through the admission where the validity of its "ambulatory" badge is checked. If he/ she does not own a badge, the data encoded during its transition at the BRS (pre admission process) are verified and completed. Then, the admission gives the patient a "hospitalization" badge concerning the data specific to the stay and will be printed on all the healthcare and examination worksheets of his/her stay.

The dietary service is informed of the arrival of the client and of the bed that is allocated to him at each moment in each of the CU in order to provide him adequate meals.

Once in possession of a valid "hospitalization" badge, the patient goes to the concerned CU. There, he/she gets medical care or a medical intervention. The medical staff indicates all the medical treatments provided during consultation on a heath care worksheet. This healthcare worksheet is finally collected by the financial service that encodes its content manually in the system for the invoicing of the services.

Once the treatments in the $\mathrm{CU}$ are finished, the patient can then be transferred internally (i.e. in another CU) to benefit from other medical treatments or interventions; these movements are still encoded manually in the system. If he/she is not transferred internally, the patient can go through other medico-technical exams. These consist in consultations with a doctor and/or x-rays and/ or providing samples for laboratory analysis (see Section B.1, process of an ambulatory patient). If he/she does not go through medico-technical exams, the patient is released from the hospital; this move must also be reported manually by the CU in the information system. All the patient moves during his/her stay at the hospital are thus traced.

At the end of the stay, all the cares are reported to the financial service that proceeds to the invoicing.

\section{B.3. Process of a patient in the emergency room (non-planned hospitalization)}

A patient in the emergency department is a patient that presents for a situation where he/she requires emergency care. His/her hospitalization has thus not been planned and the attribution of a bed in the hospital is done on a case-by-case basis.

When the patient goes to the emergency service, he/she first presents himself at the reception desk. Depending of the severity of his/her case, his/her administrative data are collected and we proceed to the creation of an "emergency" badge valid for one day only. A number is then also given to the emergency intervention he/she benefits. He/she can also be treated immediately if his/her condition is serious.

The medical treatments are then provided in the ER. The medical staff indicates the performed medical treatments on a healthcare worksheet that will document all the treatments performed during consultations. This healthcare worksheet is finally collected by the financial service that inputs its content manually in the system for the invoicing of the services.

Once the cares performed, one evaluates if the patient can leave the hospital or if he/she needs to be hospitalized. If his/her health condition allows him to leave the hospital, the medical staff registers the reasons of his/her departure. If the patient is hospitalized, the BRS is contacted in order to find an available bed in the adequate CU. The reasons for his/her hospitalization and the CU of destination are encoded. A badge "stay" is also created. The latter contains the data related to the stay and the number of the stay is equal to the number of the emergency. The patient is then transferred to the selected CU where he/she is supported the same way as above described in the process of planned hospitalization. Once the badge "stay" is created, it is physically given to the patient.

Once the patient leaves the hospital, the healthcare worksheets are brought to the financial service that proceeds to the invoicing of the cares furnished to the patient.

\section{Appendix C. Bed management at the hospital}

\section{C.1. Situation of the bed reservation in the patient flow}

In the huge majority of the cases, each patient that will be hospitalized must go through the Bed Reservation Service (BRS) for the organization of the stay. The origin of his/her hospitalization can be one of the following: the patient may be referred by his/her (family) doctor or be admitted after a consultation within the hospital, or come from another institution (transfer) or from the emergency room.

If it is not an emergency situation, the first contact between the patient and the BRS is made at the reception of the BRS. Then, before the day of admission of the patient, the contacts with the BRS are still frequent and happen most of the time by phone or mail. 
They aim to inform the patient of eventual changes or confirmations.

The day of his/her admission, the patient goes through the visitor reception center to take his/her "hospitalization badge" (required for invoicing) and complete his/her administrative data. He/she then enters in a CU where the BRS has booked a bed for him.

\section{C.2. The functions of the bed reservation service}

The functions of the BRS are:

- Reception and information of the patients;

- Planning and organization of the hospital stays;

- Management of the occupancy of the CU.

The achievement of these 3 missions requires a coordination between the functions of the organization and an exchange of information between the different stakeholders.

\section{C.2.1. Reception and information of the patients}

The reception and the information of the patients is a continuous activity subject to rhythms fluctuation linked to the flows of patients and the ease or difficulty to set the intervention or hospitalization dates. This activity can be divided in several tasks: the reception of the patient, the recording of the medical hospitalization demand, the creation of the booking materialized by the emission of a hospitalization note and the information of the patient. The objectives of this activity are: gaining the patient's trust through a visual contact and a personalized information, as well as the preparation for the admission (detection of problems in healthcare insurance for example).

\section{C.2.2. Planning and organization of hospitalization stays}

The planning and the organization of hospital stays is an activity that involves strict planning, the management of the unexpected and the constant availability in terms of communication.

Management of planned hospital stays

The objectives of this activity are the following:

- Planning of the hospital stays in function of the hospitalization notes (see Section 6). This objective relates to the booking of the medical operations and/or the medico-technical exams;

- Coordination between the different stakeholders and the patients;

- Record, classification, diffusion and achieving of the information;

- Adequate use of the material resources and management planning of an "ideal" occupancy, stable and continuous, as well as the attribution of private beds.

To be fulfilled, this function requires taking into account multiple and complex data. The bed reservation phase induces, in the context of a planned hospitalization, a process that requires the mastering of a few parameters: availability of a bed in the concerned specialization, as well as the operation schedules and the involved doctors; entry date and type of room desired by the patient, probable length of stay, prescription of preoperative exams and/or medico-technical ones. The determining parameters for choosing a hospitalization date vary from one discipline to the other. Decision criteria that can sometimes remain theoretical because the complexity of certain situations and the divergence of interests often lead to creative and negotiated solutions. Additionally, the combination of parameters may remain adjustable until the last minute so that a modification of the dates of absence of a doctor, anticipation or postposition of the intervention date, a private requirement not satisfied or a withdrawal can challenge the whole mechanism.

Management of the non-planned hospital stays

The management of the non-planned hospital stays is the answer to the demands (often performed by phone) of fast hospitalization and the search and attribution of beds for emergency hospitalizations issued by the emergency CU or the consultations. It is also the management of the internal and external patients' transfers.

As a unique coordinator and interface, the BRS must have a global vision and be able to promptly offer solutions or alternatives. In the context of a non-planned hospitalization, the control of the different parameters is depends on the real or perceived state of emergency. Also, a huge constraint comes from the strategy of some CU's to not (or as little as possible) transfer the patients to other hospitals, even when space is lacking.

\section{C.2.3. Management of the occupancy of the care units}

\section{Introduction}

The management of the occupancy of the $\mathrm{CU}$ is a continuous activity notably including the update of the CU occupancy lists, the recording of the planned movements of the patients, the allocation (in coordination with the CU) of hospital beds to new entrants, the allocation of beds for the non-planned hospitalizations and the management of the relocations of patients.

To be successfully achieved, this function requires the superposition of two different networks of activities. On the one side, an activity specific to the BRS and based on keeping a schedule of the reservations; on the other side, an activity in interaction with each 
care unit. The latter consists of establishing a forecasted vision of the occupancy of each CU. The objective is to dispose of reliable occupancy lists continuously updated. It is thus important to notice that the BRS depends on each CU to get information of the realtime bed occupancy because, without this information, it is impossible to assign beds to entering patients.

Workflow of bed management activities

The following flow describes the reception and information of the patient as well as the organization of the stay during the transit through the BRS. The patient presents himself to the BRS with his/her hospitalization demand. The BRS checks or registers the identity of the patient. If changes need to be made to the patient's data, they occur at this stage. Then, the data related to the hospitalization demand are recorded. The latter contains the concerned CU, the operation and/or the exams to be performed, the concerned doctors, etc. A hospitalization proposal is then submitted to the patient. If the patient does not accept it, a new proposal is generated, otherwise we evaluate if the patient requires surgery. If surgery is required, the intervention is included in the interventions agenda for the defined date/time. Then, if medico-technical cares are required, they are included in the patient's file and planned. The hour of arrival of the patient for his/her hospitalization, the type of room desired and the other desiderata constituting all the details of the hospitalization are then recorded. The bed reservation note is then printed and a written confirmation with the required information is given to the patient. The patient then leaves. The hospitalization demand document and the bed reservation note are then transmitted to the ANA. Thanks to these two documents, the hospitalization is written in the hospitalization agenda at the scheduled date. The hospitalization is then entered in the week schedule. If a date change is necessary, it is communicated to the patient. Otherwise, a confirmation is sent to him.

The tasks that constitute the planning and organization of the hospital stays are divided into two groups: the mid-term tasks corresponding to the planning of the CU for the planned stays, and the short-term tasks corresponding to the non-planned stays. The management of the occupancy of the CU, the third function of the BRS, is partly made during the short term phase.

- The mid-term planning takes place every Tuesday. On the basis of all the hospitalization demands and bed reservation notes, the management of the hospitalization demands of the week " $n+1$ " is performed. First of all, the printing of the entry list of week " $n+1$ " is made, which generates a weekly list of the entries for each CU. Then, the service proceeds to the sending of the hospitalization demands and of the weekly list to each CU and to the other stakeholders as well as the demand mentioning the date of the scheduled exams and the operations' program. If one or more surgeries are required, the list of the surgeries per area is printed once a week and the orthopedic material order lists are sent once per month. The process then ends;

- The short-term planning takes place every day. On the basis of the bed occupancy data of the same day per CU and of the releases that are confirmed, an updated bed occupancy list per CU is generated. In function of this, the answer to the non-planned entry demands (emergency, consultations, etc.) is determined. The daily list of entries in D-1 is then edited. Then, on the basis of the updated bed occupancy list per CU and the daily list of entries in D-1, beds are allocated to patients for the next day (D) and by CU. The attributions and the scheduled bed movements are then encoded in the system. Finally, the daily occupancy lists per CU are printed.

\section{C.3. The care units}

The administrative activities of the CU are the responsibility of the 40 Administrative Nurse Assistants (ANA) of the hospital (there is one ANA per CU).

The ANA concentrate on 3 main objectives:

- Reception of the patients and search of their file;

- Bed management with the BRS;

- Making the appointments for the medico-technical exams that the patients will have to undergo during their stay.

The proper execution of the first and third task depends, in the case of the planned hospitalizations, on the quality of the planning of the entries by the BRS. The faster an ANA receives the data concerning the next entrants, the easier he/she will find the medical files and plan the preoperative and/or medico-technical exams.

The second task requires from the ANA several daily contacts with the BRS. The latter should be informed, as quickly as possible, of the planned departures, of the extensions of stays and of the moves (for example a patient temporarily transferred to intensive care). The accuracy of the information depends on the medical organization of the CU - for example the moment (hour of the day) and the efficiency of the rooms lookup performed to determine the following departures - and the correct flow of information within the CU. The awareness of the availability of a bed can also be influenced by other parameters such as the working hours of the ANA, the cleaning of the room, the departure hour of the discharged patient, and the patient's destination (home, family, rest home, etc.).

Let's finally highlight that, for now, the work of the ANA is not formalized at all. Each ANA indeed manages the administrative work of its CU his/her own way. The different ways of working in each of the CU are due to the cared pathology, the influence of the responsible doctors imposing their way of working, and to the ability of the ANA to work with computers, etc.

\section{C.4. Workflow of the activities of the care units}

Due to the lack of standardization of the tasks of the ANA, we will limit the representation of the tasks to those generally realized in the same way in the different CUs. In order to allow a better modeling, we also have divided the work of the ANA of a CU into two 
functions: the reception and the management of the patient movements and the management of the occupancy of the CU.

- The management of the patient starts when the BRS communicates the planning of that patient's entry; this happens a week in advance (for most of the CUs). The ANA then searches his/her file and arranges appointments for the medico-technical exams that are not organized by the BRS. The moves of the patient are recorded; on the basis of these records, the admission prints daily these moves and it then re-encodes them and possibly corrects them.

- The management of the patient's stay by the CU is as follows:

- At day D-1, the list of entries for the day D is used as input and the beds are allocated to each patient. The patient's file is then retrieved and an appointment for the known medico-technical exams is made.

- At day D, the patient presents himself with his/her hospitalization demand and is welcomed. His/her entry in the hospital is registered into the information system. Then, appointments for non-planned medico-technical exams are made and the personal agenda of the patient is updated. If the patient needs to do a move (departure for the weekend, change of bed, temporary transfer, etc.) in the hospital, this move is registered. Then, when the patient gets back to its original position, this comeback is also registered so that "the move" has come to an end. If, on the contrary, the patient does not have to make any move anymore and has been taken care of, he/she is released from the hospital and his/her departure is registered. This also constitutes the end of the moves of the patient.

Finally, the management by a CU of its occupancy occurs as follows: Each Tuesday, the weekly list of planned entries serves as an input to the task of organizing and registering the planned entries. Each day, in the morning, the CU occupancy sheets are updated and a list of the beds occupancy for the CU is generated. Then, the registration of the CU's occupancy is done gradually and the beds occupancy rate of the CU is updated accordingly. The planned departures are also encoded. Finally, the answers to the demands made by the BRS for the non-planned entries are provided. On the evening, this daily loop ends up.

Most of these activities are first of all noted on an occupancy sheet before being recorded in the system. Some ANA even communicate the changes to the BRS by phone before recording.

\section{Appendix D. Paper supports of the bed management}

Both within the BRS and within the CU, paper support remains dominant. The most used documents by the BRS are:

- The medical demand of hospitalization. The data encompassed varies from a service to the other, but we mostly find:

-The reason of the hospitalization;

-The target CU;

-The entry date;

-The intervention date;

-The probable duration of hospitalization;

-The type of intervention;

-The duration of the intervention;

-The material to order;

-The degree of emergency (on a scale from 0 to 3);

-The list of preoperative exams to plan and the date an hour of the appointments taken for them;

-The postoperative exams to plan;

-The desired type of room (common ( 4 beds), 2 beds, 2 beds + TV, private);

-If the patient needs to fast prior his/her admission the entrance (yes/no);

-The medication to adapt or to suspend;

-The name of the family doctor;

-The name of the responsible doctor;

-The name of the requesting doctor.

- The hospitalization note. This document is printed after the encoding of the characteristics of the hospital stay. It indicates the administrative and medical data linked to the hospitalization.

- The hospitalization agenda is used for the scheduling of the hospital stays.

- The notification document is sent to the patient to confirm the characteristics of his/her hospitalization or to inform him from a change (e.g. of date).

- The daily occupancy list per CU. This document allows the visualization of the occupancy of the CU and is used as support for the planning and management of the moves and for the visualization of the empty beds.

- The recapitulative list of entries. The daily list is used for the information of the reception service, the emergency service and the nurse/night chef. The daily list issued to inform the providers and the CU and to prepare the hospitalizations. The recapitulative lists of entries are also used internally.

The documents used by the CU are:

- The bed occupancy panel. It allows a simple and global visualization of the occupancy of the CU and a rapid access to data (card 
mentioning gender, the administrative number, the name and the birth date of the patient).

- The bed occupancy sheet. It is the printout of the window bed occupancy displayed by the IT system. It is the main tool of the ANA on which they indicated all the moves (entries, departures, transfers, etc.) and previsions of moves in the CU. This support is often draft because of the frequent changes. It must be reprinted several times a day.

- The appointments agenda. It is used in the majority of the CU; it supports the planning of the patients' exams.

- The historic file. It includes the main data of the patients that went through the CU.

\section{References}

Belciug, S., Gorunescu, F., 2015. Improving hospital bed occupancy and resource utilization through queuing modeling and evolutionary computation. J. Biomed. Inform. 53, 261-269.

Bellifemine, F., Caire, G., Greenwood, D., 2007. Developing multi-agent systems with JADE, vol. 5 Wiley.

Bergenti, F., Poggi, A., 2010. Developing smart emergency applications with multi-agent systems. IJEHMC 1 (4), 1-13. http://dx.doi.org/10.4018/jehmc. 2010100101.

Bhat, S., Sidnal, N.S., Manvi, S.S., 2011. Agent Based Approach in Accessing Distributed Health Care Services. Springer, Berlin Heidelberg, Berlin, Heidelberg, pp. $212-221$.

Bouchoul, F., Mostefai, M., 2009. Agent-services and mobile agents for an integrated hcis. Int. J. Comput. Integr. Manuf. 22 (5), $458-471$.

Bouzguenda, L., Turki, M., 2014. Designing an architectural style for dynamic medical cross-organizational workflow management system: an approach based on agents and web services. J. Med. Syst. 38 (4), 32

Bratman, M.E., 1999. Intention, Plans, and Practical Reason. Cambridge University Press.

Braubach, L., Pokahr, A., Lamersdorf, W., 2005. Extending the capability concept for flexible bdi agent modularization. In: International Workshop on Programming Multi-Agent Systems. Springer, pp. 139-155.

Braubach, L., Pokahr, A., Lamersdorf, W., 2014. Negotiation-based patient scheduling in hospitals - reengineering message-based interactions with services. In: Iantovics, B., Kountchev, R. (Eds.), Advanced Intelligent Computational Technologies and Decision Support Systems. Studies in Computational Intelligence, vol. 486. Springer, pp. 107-121.

Braubach, L., Lamersdorf, W., Pokahr, A., 2003. Jadex: Implementing a bdi-infrastructure for jade agents.

Bresciani, P., Perini, A., Giorgini, P., Giunchiglia, F., Mylopoulos, J., 2004. Tropos: an agent-oriented software development methodology. Auton. Agent. Multi-Agent Syst. 8 (3), 203-236.

Cardoso, L., Marins, F., Portela, F., Santos, M., Abelha, A., Machado, J., 2014. The next generation of interoperability agents in healthcare. Int. J. Environ. Res. Public Health 11 (5), 5349-5371.

Cardoso, L., Marins, F., Portela, F., Abelha, A., Machado, J., 2014. Healthcare interoperability through intelligent agent technology. Proc. Technol. 16, 1334-1341.

Casali, A., Godo, L., Sierra, C., 2011. A graded BDI agent model to represent and reason about preferences. Artif. Intell. 175 (7-8), $1468-1478$.

Castro, J., Kolp, M., Mylopoulos, J., 2002. Towards requirements-driven information systems engineering: the tropos project. Inf. Syst. 27 (6), $365-389$.

Castro, J., Kolp, M., Mylopoulos, J., 2013. A requirements-driven development methodology. In: Seminal Contributions to Information Systems Engineering, 25 Years of CAiSE, pp. 265-280.

Chen, J., Wang, P., Huang, Y., Yen, H., 2012. Applying ontology techniques to develop a medication history search and alert system in department of nuclear medicine. J. Med. Syst. 36 (2), 737-746.

Chennaoui, A., Paquet, M., 2014. Multi-agent decision-making support model for the management of pre-hospital emergency services. Int. J. Mach. Learn. Comput. 4 (2), 198.

Chinosi, M., Trombetta, A., 2012. Bpmn: an introduction to the standard. Comput. Stand. Interfaces 34 (1), $124-134$.

Chung, L., Nixon, B., Yu, E., Mylopoulos, J., 2000. Non-Functional Requirements in Software Engineering. Kluwer Publishing.

Cresswell, K.M., Bates, D.W., Sheikh, A., 2017. Ten key considerations for the successful optimization of large-scale health information technology. JAMIA 24 (1), $182-187$.

Darwin, P.B., Kozlowski, P., 2013. Angular JS Web Application Development. Packt Publishing.

Davis, M.C., Challenger, R., Jayewardene, D.N., Clegg, C.W., 2014. Advancing socio-technical systems thinking: a call for bravery. Appl. Ergon. 45 (2), $171-180$.

De Giacomo, G., Lespérance, Y., Muise, C., 2012. On supervising agents in situation-determined ConGolog. In: Proceedings of the 11th International Conference on Autonomous Agents and Multiagent Systems-Volume 2, International Foundation for Autonomous Agents and Multiagent Systems, pp. 1031-1038.http://www. cse.yorku.ca/lesperan/papers/AAMAS12.pdf.

F.F. for Intelligent Physical Agents, 2002. Fipa quality of service ontology specification, Doc.No. SC00094A. FIPA ( http://www.fipa.org).

Fox, J., Beveridge, M., Glasspool, D., 2003. Understanding intelligent agents: analysis and synthesis. AI Commun. 16 (3), $139-152$.

Guedes, G.T., Vicari, R.M., 2009. Applying auml and uml 2 in the multi-agent systems project. In: Proceedings of the ER 2009 Workshops on Advances in Conceptual Modeling - Challenging Perspectives, ER ’09, Springer-Verlag, Berlin, Heidelberg, pp. 106-115.

Gund, M., Andhalkar, S., Patil, D., Wadhai, V., et al., 2011. An intelligent architecture for multi-agent based m-health care system. Int. J. 1-5.

Hall, R., 2012. Bed assignment and bed management. In: Handbook of Healthcare System Scheduling. Springer, pp. 177-200.

Hevner, A.R., March, S.T., Park, J., Ram, S., 2004. Design science in information systems research. MIS Q. 28 (1), 75-105. URLhttp://misq.org/design-science-ininformation-systems-research.html.

Hogg, L., Jennings, N.R., 2001. Socially intelligent reasoning for autonomous agents. IEEE Trans Systems Man Cybernetics, Part A 31 (5), $381-393$.

Isern, D., Moreno, A., 2016. A systematic literature review of agents applied in healthcare. J. Med. Syst. 40 (2), 43:1-43:14.

Isern, D., Sánchez, D., Moreno, A., 2010. Agents applied in health care: A review. Int. J. Med. Inform. 79 (3), 145-166.

Isern, D., Sánchez, D., Moreno, A., 2012. Ontology-driven execution of clinical guidelines. Comput. Methods Programs Biomed. 107 (2), $122-139$.

Kolp, M., Wautelet, Y., 2015. Human organizational patterns applied to collaborative learning software systems. Comput. Hum. Behav. 51, $742-751$.

Kolp, M., Faulkner, S., Wautelet, Y., 2008. Social structure based design patterns for agent-oriented software engineering. IJIIT 4 (2), 1-23. http://dx.doi.org/10.4018/ jiit.2008040101.

Kolp, M., Wautelet, Y., Faulkner, S., 2011. Social-centric design of multi-agent architectures. In: Yu, E., Giorgini, P., Maiden, N., Mylopoulos, J. (Eds.), Social Modeling for Requirements Engineering. MIT Press.

Liu, C., Chung, Y., Chiang, T., Chen, T., Wang, S., 2012. A mobile agent approach for secure integrated medical information systems. J. Med. Syst. 36 (5), $2731-2741$.

Maloney, C.G., Wolfe, D., Gesteland, P.H., Hales, J.W., Nkoy, F.L., 2007. A tool for improving patient discharge process and hospital communication practices: the patient tracker. In: AMIA Annual Symposium Proceedings, vol. 2007, American Medical Informatics Association, p. 493.

Nguyen, M.T., Fuhrer, P., Pasquier-Rocha, J., 2009. Enhancing e-health information systems with agent technology. Int. J. Telemedicine Appl. 2009, 1:1-1:13. http:// dx.doi.org/10.1155/2009/279091.

OMG, 2013. Business process model and notation (bpmn). version 2.0.1, Tech. rep., Object Management Group.

OMG, 2015. Omg unified modeling languageTM (omg uml). version 2.5, Tech. rep., Object Management Group.

Payne, T.H., Bates, D.W., Berner, E.S., Bernstam, E.V., Covvey, H.D., Frisse, M.E., Graf, T., Greenes, R.A., Hoffer, E.P., Kuperman, G.J., Lehmann, H.P., Liang, L., Middleton, B., Omenn, G.S., Ozbolt, J.G., 2013. Healthcare information technology and economics. JAMIA 20 (2), $212-217$.

Peffers, K., Tuunanen, T., Rothenberger, M., Chatterjee, S., 2007. A design science research methodology for information systems research. J. Manage. Inf. Syst. 24 (3), 45-77. http://dx.doi.org/10.2753/MIS0742-1222240302. 
Peffers, K., Tuunanen, T., Rothenberger, M.A., Chatterjee, S., 2008. A design science research methodology for information systems research. J. Manage. Inf. Syst. 24 (3), 45-77. URLhttp://www.jmis-web.org/articles/765.

Pickering, B.W., Litell, J.M., Herasevich, V., Gajic, O., 2012. Clinical review: the hospital of the future-building intelligent environments to facilitate safe and effective acute care delivery. Crit. Care 16 (2), 1.

Pokahr, A., Braubach, L., Haubeck, C., Ladiges, J., 2014. Programming BDI agents with pure java. In: Müller, J.P., Weyrich, M., Bazzan A.L.C., (Eds.), Multiagent System Technologies - 12th German Conference, MATES 2014, Stuttgart, Germany, September 23-25, 2014. Proceedings, vol. 8732 of LNCS, Springer, pp. 216-233.

Poslad, S., 2007. Specifying protocols for multi-agent systems interaction, ACM Trans. Auton. Adapt. Syst. 2(4). doi: http://dx.doi.org/10.1145/1293731.1293735.

Rao, A.S., Georgeff, M.P., 1995. BDI agents: from theory to practice. In: Lesser, V.R., Gasser, L. (Eds.), Proceedings of the First International Conference on Multiagent Systems, June 12-14, 1995, San Francisco, California, USA. The MIT Press, pp. 312-319.

Sayyad-Shirabad, J., Wilk, S., Michalowski, W., Farion, K., 2012. Implementing an integrative multi-agent clinical decision support system with open source software. J. Med. Syst. 36 (1), 123-137. http://dx.doi.org/10.1007/s10916-010-9452-9.

Shi, P., Chou, M.C., Dai, J., Ding, D., Sim, J., 2015. Models and insights for hospital inpatient operations: time-dependent ed boarding time. Manage. Sci. 62 (1), 1-28.

Simon, H.A., 1996. The Sciences of the Artificial, third ed. MIT Press, Cambridge, MA, USA.

Stephen, A., 2008. White, Derek Miers, BPMN Modeling and Reference Guide. Future Strategies Inc., Lighthouse Pt, FL.

Taboada, M., Cabrera, E., Iglesias, M.L., Epelde, F., Luque, E., 2011. An agent-based decision support system for hospitals emergency departments. In: Sato, M.,

Matsuoka, S., Sloot, P.M.A., van Albada, G.D., Dongarra J., (Eds.), Proceedings of the International Conference on Computational Science, ICCS 2011, Nanyang Technological University, Singapore, 1-3 June, 2011, vol. 4 of Procedia Computer Science, Elsevier, pp. 1870-1879.

Tamburri, D.A., Lago, P., van Vliet, H., 2013. Organizational social structures for software engineering. ACM Comput. Surv. 46 (1), 3:1-3:35.

Ulrich, K.T., 2011. Design: Creation of Artifacts in Society. University of Pennsylvania. URLhttp://opim.wharton.upenn.edu/ulrich/designbook.html.

van Deursen, N., Buchanan, W.J., Duff, A., 2013. Monitoring information security risks within health care. Comput. Secur. $37,31-45$.

Varshney, U., 2009. Pervasive Healthcare Computing: EMR/EHR, Wireless and Health Monitoring, Incorporated, 1st Edition. Springer Publishing Company.

Wautelet, Y., Kolp, M., 2016. Business and model-driven development of BDI multi-agent systems. Neurocomputing 182, 304-321. http://dx.doi.org/10.1016/j. neucom.2015.12.022.

Wautelet, Y., Heng, S., Kolp, M., Penserini, L., Poelmans, S., 2016. Designing an MOOC as an agent-platform aggregating heterogeneous virtual learning environments. Behav. IT 35 (11), 980-997.

Wautelet, Y., Kolp, M., Heng, S., 2017. Bed reservation automation: Analysis and design for a university hospital, Technical Report.http://hdl.handle.net/2078.1/ 179512.

Wooldridge, M.J., 2009. An Introduction to MultiAgent Systems, second ed. Wiley.

Yen, P., Bakken, S., 2012. Review of health information technology usability study methodologies. JAMIA 19 (3), $413-422$.

Yu, E.S.K., 2009. Social modeling and i*. In: Conceptual Modeling: Foundations and Applications - Essays in Honor of John Mylopoulos, pp. 99-121.

Yu, E., Giorgini, P., Maiden, N., Mylopoulos, J., 2011. Social Modeling for Requirements Engineering. MIT Press.

Zhang, X., Xu, H., Shrestha, B., 2009. Building a health care multi-agent simulation system with role-based modeling. Multi-Agent Systems for Healthcare Simulation and Modeling: Applications for System Improvement: Applications for System Improvement, 90. 